\title{
SEMIFLOWS FOR DIFFERENTIAL EQUATIONS WITH LOCALLY BOUNDED DELAY \\ ON SOLUTION MANIFOLDS IN THE SPACE $C^{1}\left((-\infty, 0], \mathbb{R}^{n}\right)$
}

\author{
HANS-OTto Walther
}

\begin{abstract}
We construct a semiflow of continuously differentiable solution operators for delay differential equations $x^{\prime}(t)=f\left(x_{t}\right)$ with $f$ defined on an open subset of the Fréchet space $C^{1}=C^{1}\left((-\infty, 0], \mathbb{R}^{n}\right)$. This space has the advantage that it contains all histories $x_{t}=x(t+\cdot), t \in \mathbb{R}$, of every possible entire solution of the delay differential equation, in contrast to a Banach space of maps $(-\infty, 0] \rightarrow \mathbb{R}^{n}$ whose norm would impose growth conditions at $-\infty$. The semiflow lives on the set $X_{f}=\left\{\phi \in U: \phi^{\prime}(0)=f(\phi)\right\}$ which is a submanifold of finite codimension in $C^{1}$. The hypotheses are that the functional $f$ is continuously differentiable (in the Michal-Bastiani sense) and that the derivatives have a mild extension property. The result applies to autonomous differential equations with state-dependent delay which may be unbounded but which is locally bounded. The case of constant bounded delay, distributed or not, is included.
\end{abstract}

\section{Introduction}

An autonomous delay differential equation has the form

$$
x^{\prime}(t)=f\left(x_{t}\right)
$$

with a functional $f: U \rightarrow \mathbb{R}^{n}$, where $U \subset\left(\mathbb{R}^{n}\right)^{I}$ is a set of maps $I \rightarrow \mathbb{R}^{n}$ defined on a closed interval $I \subset \mathbb{R}$ of positive length with $\max I=0$. A solution on an interval $J \subset \mathbb{R}$ is a map $x: I+J \rightarrow \mathbb{R}^{n}$ so that $\left.x\right|_{J}$ is differentiable, all segments,

2010 Mathematics Subject Classification. 34K05, 37L05.

Key words and phrases. Delay differential equation; state-dependent delay; unbounded delay; Fréchet space. 
or histories, $x_{t}: I \ni s \mapsto x(s+t) \in \mathbb{R}^{n}, t \in J$, belong to $U$, and equation (1.1) holds for all $t \in J$, with the right derivative of $x$ at $t=\min J$ in case $J$ has a minimum (or, with the derivative of $\left.x\right|_{J}$ on the left-hand side).

In case $I=[-r, 0], r>0, U \subset C\left(I, \mathbb{R}^{n}\right)$ open, and $f$ locally Lipschitz continuous the initial value problem (IVP)

$$
x^{\prime}(t)=f\left(x_{t}\right) \quad \text { for } t>0, \quad x_{0}=\phi \in U \subset C\left([-r, 0], \mathbb{R}^{n}\right)
$$

has a unique maximal solution $x=x^{\phi}$ on some interval $J=\left[0, t_{\phi}\right)$, and the solution operators $\phi \mapsto x_{t}^{\phi}, 0 \leq t<t_{\phi}$, define a continuous semiflow on $U$. For $f$ continuously differentiable the solution operators are continuously differentiable, see [2] and compare [5]. This by now familiar theory applies to examples like

$$
x^{\prime}(t)=g(x(t), x(t-r)) \quad \text { or } \quad x^{\prime}(t)=\int_{-r}^{0} g(x(t+s)) d \mu(s)
$$

where the delay is invariant, that is, does not depend neither on the argument $t$ nor on the state $\phi=x_{t}$.

For case $I=(-\infty, 0]$ and equations with unbounded invariant delay, results on well-posed IVPs in suitable Banach spaces of continuous functions $(-\infty, 0] \rightarrow$ $\mathbb{R}^{n}$ may be found in [8] and in [1].

All of these do not cover equations with a variable, state-dependent delay, like for example

$$
x^{\prime}(t)=g\left(x\left(t-d\left(x_{t}\right)\right)\right)
$$

with a nonconstant delay functional $d: C\left([-r, 0], \mathbb{R}^{n}\right) \supset U \rightarrow[0, r]$. The reason for this may be seen in the fact that the evaluation map

$$
C\left([-r, 0], \mathbb{R}^{n}\right) \times[-r, 0] \ni(\phi, s) \mapsto \phi(s) \in \mathbb{R}^{n}
$$

is not locally Lipschitz continuous [14]. A theory which applies to equations with bounded state-dependent delay and yields continuously differentiable solution operators was developed in $[14,15]$. The main result in [14] considers equation (1.1) for a continuously differentiable functional $f: U \rightarrow \mathbb{R}^{n}, U$ an open subset of the Banach space $C^{1}\left([-r, 0], \mathbb{R}^{n}\right)$, and establishes a continuous semiflow of continuously differentiable solution operators for equation (1.1) under a mild additional smoothness hypothesis, which requires that

[e] every derivative $D f(\phi), \phi \in U$, extends to a linear map

$$
D_{\mathrm{e}} f(\phi): C\left([-r, 0], \mathbb{R}^{n}\right) \rightarrow \mathbb{R}^{n}
$$

and the map

$$
U \times C\left([-r, 0], \mathbb{R}^{n}\right) \ni(\phi, \chi) \mapsto D_{\mathrm{e}} f(\phi) \chi \in \mathbb{R}^{n}
$$

is continuous. 
This is a variant of the property of being almost Fréchet differentiable from [9], designed for the application to differential equations with bounded state-dependent delay. The semiflow from [14] lives on the solution manifold

$$
X_{f}=\left\{\phi \in U: \phi^{\prime}(0)=f(\phi)\right\}
$$

which is indeed a continuously differentiable submanifold of codimension $n$ in $C^{1}\left([-r, 0], \mathbb{R}^{n}\right)$.

In [16] the result from [14] is used to obtain analogous results for a class of equations which involve unbounded state-dependent delay, like for example equation (1.2) with a delay functional $d: U \rightarrow[0, \infty)$ which may be unbounded but is locally bounded - with respect to a topology on $U$. In [16] equation (1.1) is studied for a map $f$ defined on an open subset $U$ of a Banach space $B_{a}^{1}, a>0$, of continuously differentiable maps $(-\infty, 0] \rightarrow \mathbb{R}^{n}$ which satisfy

$$
e^{a t} \phi(t) \rightarrow 0 \text { and } \quad e^{a t} \phi^{\prime}(t) \rightarrow 0 \quad \text { as } t \rightarrow-\infty .
$$

The property that such a map $f: B_{a}^{1} \supset U \rightarrow \mathbb{R}^{n}$ is of locally bounded delay means that

[lbd ] for every $\phi \in U$ there are a neighbourhood $V \subset U$ and $r>0$ such that for all $\chi, \psi$ in $V$ with $\chi(t)=\psi(t)$, for all $t \in[-r, 0]$, we have $f(\chi)=f(\psi)$.

A further extension in [17] concerns the construction of an evolutionary system (or, a process) of continuously differentiable solution operators for a nonautonomous delay differential equation

$$
x^{\prime}(t)=f\left(t, x_{t}\right)
$$

with a continuously differentiable map $f: \mathbb{R} \times B_{a}^{1} \supset V \rightarrow \mathbb{R}^{n}$ which satisfies nonautonomous versions of the properties [e] and [lbd]. Applications include, for example, Volterra integro-differential equations of the form

$$
x^{\prime}(t)=\int_{0}^{t} k(t, s) g(x(s)) d s
$$

where the time-dependent delay at time $t$ is bounded by $t$, and equations with proportional delay like the pantograph equation

$$
x^{\prime}(t)=a x(\lambda t)+b x(t)
$$

with $0<\lambda<1$, where the time-dependent delay is given by $d(t)=(1-\lambda) t$, because of $x(\lambda t)=x(t-d(t))$.

The solution operators from [17] are defined on open subsets of continuously differentiable submanifolds of finite codimension in the Banach space $B_{a}^{1}$.

Working in a Banach space like $B_{a}^{1}, a>0$, which is defined by a growth condition at $-\infty$, has a shortcoming, though. Linearization of the semiflows from [16] 
at equilibria yields autonomous linear differential equations with bounded invariant delay. For almost all solutions $\lambda \in \mathbb{C}$ of the characteristic equation associated to such a linear delay differential equation the nontrivial entire solutions (solutions on $J=\mathbb{R}$, in the former terminology)

$$
x: \mathbb{R} \ni t \mapsto \operatorname{Re} e^{\lambda t} c \in \mathbb{R}^{n}, \quad c \in \mathbb{C}^{n},
$$

grow too fast at $-\infty$ for having segments $x_{t}$ in the space $B_{a}^{1}$. This means, in particular, that the spectrum of the generator of the linearized semiflow is no longer given by the characteristic equation, which results from an Ansatz with exponential solutions.

A state space which contains all segments $x_{t}:(-\infty, 0] \rightarrow \mathbb{R}^{n}, t \in \mathbb{R}$, of all continuously differentiable solutions of an autonomous differential equation with locally bounded delay together with all segments of all entire solutions of its linearizations must not be restricted by growth or integrability conditions at $-\infty$. This suggests to try the vector space $C^{1}=C^{1}\left((-\infty, 0], \mathbb{R}^{n}\right)$, which equipped with the topology of uniform convergence of maps and their derivatives on compact sets, is a Fréchet space, that is, a complete topological vector space whose topology is given by a sequence of seminorms $|\cdot|_{j}, j \in \mathbb{N}$, which is separating $\left(\lim _{k \rightarrow \infty} x_{k}=x\right.$ if and only if $\lim _{k \rightarrow \infty}\left|x_{k}-x\right|_{j}=0$ for every $j \in \mathbb{N}$, and $x=0$ if and only if $|x|_{j}=0$ for all $j \in \mathbb{N}$ ).

Let us recall here that continuous differentiability in Banach spaces does not generalize in a canonical way to topological vector spaces. In the sequel continuous differentiability of a map $f: V \supset U \rightarrow W, V$ and $W$ topological vector spaces and $U \subset V$ open, is always understood in the sense of Michal and Bastiani, which means the following: The tangent vector of a continuous curve $c: I \rightarrow V, I \subset \mathbb{R}$ an interval of positive length, at $t \in I$ is defined by

$$
c^{\prime}(t)=\lim _{0 \neq h \rightarrow 0} \frac{1}{h}(c(t+h)-c(t)) \in V
$$

provided this limit exists. The curve is called continuously differentiable if it has tangent vectors everywhere and if the map $I \ni t \mapsto c^{\prime}(t) \in V$ is continuous. For a continuous map $f: V \supset U \rightarrow W$ as above and for $u \in U$ and $v \in V$ with $u+(-\varepsilon, \varepsilon) v \subset U$ for some $\varepsilon>0$ the directional derivative $D f(u) v \in W$ is defined by the tangent vector of the curve $(-\varepsilon, \varepsilon) \ni t \mapsto f(u+t v) \in W$ at $t=0$ provided the latter exists. The map $f$ is called continuously differentiable if all directional derivatives $D f(u) v, u \in U$ and $v \in V$, exist and if the map

$$
U \times V \ni(u, v) \mapsto D f(u) v \in W
$$

is continuous. 
This notion, obviously weaker than familiar continuous differentiability of maps in Banach spaces, avoids the choice of a topology on the vector space of linear continuous maps $V \rightarrow W$. For more on this, consult e.g. [13], [3], [6].

Let $C=C\left((-\infty, 0], \mathbb{R}^{n}\right)$ denote the Fréchet space of continuous maps $(-\infty, 0]$ $\rightarrow \mathbb{R}^{n}$, with the topology of uniform convergence on compact sets.

The present paper deals with equation (1.1) for continuously differentiable functionals $f$ on open subsets $U \subset C^{1}$ so that the analogue (e) of the extension property $[\mathrm{e}]$ holds where $C^{1}\left([-r, 0], \mathbb{R}^{n}\right)$ and $C\left([-r, 0], \mathbb{R}^{n}\right)$ are replaced by $C^{1}$ and $C$, respectively. The main results are that, analogously to the results in [14], [16], the solution manifold $X_{f}$ is a continuously differentiable submanifold of codimension $n$ in $C^{1}$ (Proposition 2.3 below) and the maximal solutions of the IVPs

$$
x^{\prime}(t)=f\left(x_{t}\right) \quad \text { for } t>0, \quad x_{0}=\phi \in X_{f}
$$

define a continuous semiflow $\Sigma_{f}$ of continuously differentiable solution operators on $X_{f}$ (Proposition 5.2, Corollary 5.4). Proposition 6.1 shows that the derivatives

$$
D_{2} \Sigma_{f}(t, \phi): T_{\phi} X_{f} \rightarrow T_{\Sigma(t, \phi)} X_{f}
$$

of the solution operators are given by variational equations

$$
v^{\prime}(t)=\operatorname{Df}\left(\Sigma_{f}(t, \phi)\right) v_{t}
$$

In a forthcoming paper [18] we shall obtain local invariant manifolds at stationary points of the semiflow $\Sigma_{f}$.

At the beginning of Section 2 below it is shown how to verify property (e) for a simple but nevertheless typical example of the form (1.2). When written in the form (1.1) this example is of locally bounded delay (with the space $B_{a}^{1}$ in condition [lbd] replaced by $C^{1}$ ). This is not merely a coincidence - being of locally bounded delay follows from continuous differentiability, see Proposition 1.1 at the end of this introduction. Equations which involve integration over $(-\infty, 0]$, like for example

$$
x^{\prime}(t)=\int_{-\infty}^{0} h\left(x_{t}, x(t+s)\right) d s
$$

or infinite series as in

$$
x^{\prime}(t)=-\sum_{k=1}^{\infty} 2^{-k} x(t-k-r(x(t))), \quad r: \mathbb{R} \rightarrow[0, \infty) \text { bounded, }
$$

will in general escape the approach presented here. Such examples require state spaces which are restricted by the condition that the term on the right-hand side of the differential equation makes sense. The results below do not include statements about partial derivatives $\partial_{t} \Sigma_{f}(t, \phi)$, in contrast to [14], [15] about equations with bounded delay. It is easy to see that in the present case such 
partial derivatives may exist only at initial data $\phi \in X_{f} \subset C^{1}$ which are twice continuously differentiable.

Local solutions of IVPs are obtained by means of a result due to Glöckner [4], on uniform contractions whose fixed points are continuously differentiable with respect to a parameter in a topological vector space. The special case needed here, with parameters in a Fréchet space, is stated in the appendix, Section 7, as Theorem 7.2. This theorem also yields a simple Implicit Function Theorem for maps $F \times B \supset U \rightarrow B, F$ a Fréchet space and $B$ a Banach space, which is stated as Theorem 7.3. The case of Theorem 7.3 with $\operatorname{dim} B<\infty$, which helps to show that the set $X_{f}$ is a submanifold, is part of a much more general result of Glöckner [3]. The proofs of Theorems 7.2 and 7.3 are included for convenience and simplicity.

The approach in Sections 2-6 is similar to the Banach space case studied in [14], with modifications most of which are required by the lack of norms and by the different notions of continuous differentiability. The hypothesis (e) is used in the proof that $X_{f}$ is a continuously differentiable submanifold (Proposition 2.3) and in the proof of the estimate in Proposition 3.4 with a norm on the right-hand side which does not involve derivatives. This is crucial in order to obtain the Lipschitz estimate of Proposition 4.1, which in turn yields a contraction. The construction of the semiflow in Section 5 from the local results in Section 4 is standard and included for convenience.

For earlier work on differential equations with unbounded delay and solution segments in topological vector spaces, see [11], [12].

Preliminaries. For basic facts about topological vector spaces see [10]. The facts from calculus in Fréchet spaces, including the Riemann integral for continuous maps $[a, b] \rightarrow F$ into a Fréchet space and up to Fréchet manifolds, which are freely used in Sections 2-7 below, are taken from Sections I.1-I.4 in [6].

Products of topological vector spaces are always equipped with the product topology. The product $F \times G$ of Fréchet spaces is a Fréchet space.

For maps $f: U \rightarrow Z, V, W, Z$ topological vector spaces and $U \subset V \times W$ open, partial derivatives are defined in the usual way. For example, $D_{1} f(v, w): V \rightarrow Z$ is given by

$$
D_{1} f(v, w) \widehat{v}=\lim _{0 \neq h \rightarrow 0} \frac{1}{h}(f(v+h \widehat{v}, w)-f(v)) .
$$

The tangent cone of a set $M \subset F, F$ a Fréchet space, at $x \in M$ is the set $T_{x} M$ of all tangent vectors $v=c^{\prime}(0)$ of continuously differentiable curves $c: I \rightarrow F$ with $I$ open, $0 \in I, c(0)=x, c(I) \subset M$.

The following Fréchet spaces are used in the sequel: For $n \in \mathbb{N}$ and $T \in \mathbb{R}$, $C_{T}=C\left((-\infty, T], \mathbb{R}^{n}\right)$ denotes the Fréchet space of continuous maps $(-\infty, T] \rightarrow$ $\mathbb{R}^{n}$ with the seminorms given by $|\phi|_{T, j}=\max _{T-j \leq t \leq T}|\phi(t)|, \phi \in C_{T}$ and $j \in \mathbb{N}$, 
which define the topology of uniform convergence on compact sets, and $C_{T}^{1}=$ $C^{1}\left((-\infty, T], \mathbb{R}^{n}\right)$ is the Fréchet space of continuously differentiable maps $(-\infty, T]$ $\rightarrow \mathbb{R}^{n}$ with $|\phi|_{1, T, j}=|\phi|_{T, j}+\left|\phi^{\prime}\right|_{T, j}$.

Analogously, $C_{\infty}=C\left(\mathbb{R}, \mathbb{R}^{n}\right)$ denotes the Fréchet space of continuous maps $\mathbb{R} \rightarrow \mathbb{R}^{n}$ with $|\phi|_{\infty, j}=\max _{-j \leq t \leq j}|\phi(t)|$, and $C_{\infty}^{1}=C^{1}\left(\mathbb{R}, \mathbb{R}^{n}\right)$ denotes the Fréchet space of continuously differentiable maps $\mathbb{R} \rightarrow \mathbb{R}^{n}$ with $|\phi|_{1, \infty, j}=|\phi|_{\infty, j}+\left|\phi^{\prime}\right|_{\infty, j}$. The differentiation map $\partial_{T}: C_{T}^{1} \ni \phi \mapsto \phi^{\prime} \in C_{T}$ is linear and continuous.

It will be convenient to use $C=C_{0}$ and $C^{1}=C_{0}^{1}$ and to abbreviate $|\cdot|_{j}=$ $|\cdot|_{0, j},|\cdot|_{1, j}=|\cdot|_{1,0, j}, \partial=\partial_{0}$. The convex sets

$$
\left\{\phi \in C:|\phi|_{j}<\frac{1}{j}\right\}, \quad j \in \mathbb{N},
$$

form a neighbourhood base at $0 \in C$, and the convex sets

$$
\left\{\phi \in C^{1}:|\phi|_{1, j}<\frac{1}{j}\right\}, \quad j \in \mathbb{N},
$$

form a neighbourhood base at $0 \in C^{1}$.

Every Banach space $B$ over $\mathbb{R}$, with norm $|\cdot|$, is a Fréchet space with $|\cdot|_{j}=|\cdot|$ for $j \in \mathbb{N}$. For Banach spaces $B, E$ the Banach space of linear continuous maps $B \rightarrow E$ is denoted by $L_{\mathrm{c}}(B, E)$.

The following Banach spaces occur in the sequel: For $n \in \mathbb{N}$ and real numbers $S<T$, the space $C_{S T}=C\left([S, T], \mathbb{R}^{n}\right)$ of continuous maps $[S, T] \rightarrow \mathbb{R}^{n}$ with the norm given by $|\phi|_{S T}=\max _{S \leq t \leq T}|\phi(t)|$, and the space $C_{S T}^{1}=C^{1}\left([S, T], \mathbb{R}^{n}\right)$ of continuously differentiable maps $[S, T] \rightarrow \mathbb{R}^{n}$ with the norm given by $|\phi|_{1, S T}=$ $|\phi|_{S T}+\left|\phi^{\prime}\right|_{S T}$.

In case $S=0<T, C_{0 T, 0}$ denotes the closed subspace $\left\{\phi \in C_{0 T}: \phi(0)=0\right\}$, and $C_{0 T, 0}^{1}$ denotes the closed subspace $\left\{\phi \in C_{0 T}^{1}: \phi(0)=0=\phi^{\prime}(0)\right\}$.

Proposition 1.1. Each continuously differentiable map $f: C^{1} \supset U \rightarrow \mathbb{R}^{n}$ is of locally bounded delay in the sense that for every $\phi \in U$ there are a neighbourhood $V \subset U$ and $r>0$ such that for all $\chi, \psi$ in $V$ with

$$
\chi(t)=\psi(t) \quad \text { for all } t \in[-r, 0]
$$

we have $f(\chi)=f(\psi)$.

Proof. For $j \in \mathbb{N}$ set $V_{j}=\left\{\eta \in C^{1}:|\eta|_{1, j}<1 / j\right\}$. Assume the assertion is false. Then there exists $\phi \in U$ such that for every $j \in \mathbb{N}$ there are $\chi_{j}, \psi_{j}$ in $\left(\phi+V_{j}\right) \cap U$ with $\chi_{j}(t)=\psi_{j}(t)$ for $-j \leq t \leq 0$ and $0 \neq f\left(\chi_{j}\right)-f\left(\psi_{j}\right)$. There exists $j_{\phi} \in \mathbb{N}$ so that $\phi+V_{j} \subset U$ for all integers $j \geq j_{\phi}$. For these integers $j$,

$$
0 \neq f\left(\chi_{j}\right)-f\left(\psi_{j}\right)=\int_{0}^{1} D f\left(\psi_{j}+s\left(\chi_{j}-\psi_{j}\right)\right)\left[\chi_{j}-\psi_{j}\right] d s,
$$


and it follows that for some $s_{j} \in[0,1]$,

$$
0 \neq D f\left(\psi_{j}+s_{j}\left(\chi_{j}-\psi_{j}\right)\right)\left[\chi_{j}-\psi_{j}\right] .
$$

For integers $j \geq j_{\phi}$ define $c_{j}>0$ by

$$
c_{j}\left|D f\left(\psi_{j}+s_{j}\left(\chi_{j}-\psi_{j}\right)\right)\left[\chi_{j}-\psi_{j}\right]\right|=1 .
$$

Observe that $\phi+V_{j} \ni \psi_{j}+s_{j}\left(\chi_{j}-\psi_{j}\right) \rightarrow \phi$ as $j \rightarrow \infty$. Also, $c_{j}\left[\chi_{j}-\psi_{j}\right] \rightarrow 0$ as $j \rightarrow \infty$ because given $k \in \mathbb{N}$ and $j \geq \max \left\{j_{\phi}, k\right\}, c_{j}\left[\chi_{j}-\psi_{j}\right](t)=0$ on $[-j, 0] \supset[-k, 0]$, hence $\left|c_{j}\left[\chi_{j}-\psi_{j}\right]\right|_{1, k}=0$. By continuity, $|D f(\phi) 0|=1$, contradicting linearity.

\section{The extension property and the solution manifold}

In order to rewrite examples like equation (1.2) in the general form of equation (1.1) evaluation maps are convenient. With the numerical evaluation map

$$
\text { ev: }\left(\mathbb{R}^{n}\right)^{(-\infty, 0]} \times(-\infty, 0] \ni(\phi, t) \mapsto \phi(t) \in \mathbb{R}^{n}
$$

in case $n=1$ the right-hand side of $(1.2)$ is given by $(1.1)$ for $U=\mathbb{R}^{(-\infty, 0]}$ and

$$
f(\phi)=g(\operatorname{ev}(\phi,-d(\phi)))=g \circ \mathrm{ev} \circ(\mathrm{id} \times(-d))(\phi) .
$$

Proposition 2.1. ev $0=\left.\mathrm{ev}\right|_{C \times(-\infty, 0]}$ is continuous, and $\mathrm{ev}_{1}=\left.\mathrm{ev}\right|_{C^{1} \times(-\infty, 0)}$ is continuously differentiable with

$$
D \operatorname{ev}_{1}(\phi, t)(\widehat{\phi}, \widehat{t})=\widehat{\phi}(t)+\widehat{t} \phi^{\prime}(t) .
$$

Proof. 1. (On evo) Suppose $\phi_{k} \rightarrow \phi$ in $C$ and $t_{k} \rightarrow t$ in $(-\infty, 0]$ as $\mathbb{N} \ni k \rightarrow \infty$. There exists an integer $j \in \mathbb{N}$ with $-j<t_{k}$ for all $k \in \mathbb{N}$. Use

$$
\begin{aligned}
\left|\operatorname{ev}\left(\phi_{k}, t_{k}\right)-\operatorname{ev}(\phi, t)\right| & =\left|\phi_{k}\left(t_{k}\right)-\phi(t)\right| \\
& \leq\left|\phi_{k}\left(t_{k}\right)-\phi\left(t_{k}\right)\right|+\left|\phi\left(t_{k}\right)-\phi(t)\right| \\
& \leq\left|\phi_{k}-\phi\right|_{0, j}+\left|\phi\left(t_{k}\right)-\phi(t)\right|
\end{aligned}
$$

and $\left|\phi_{k}-\phi\right|_{0, j} \rightarrow 0$ for $k \rightarrow \infty$ and the continuity of $\phi$ at $t$.

2. $\left(\mathrm{On} \mathrm{ev}_{1}\right)$ Each map ev $(\cdot, t), t \leq 0$, is linear. This implies that for every $\phi \in C^{1}, t \leq 0$ and $\widehat{\phi} \in C^{1}$ the directional derivative $D \operatorname{ev}_{1}(\phi, t) \widehat{\phi} \in \mathbb{R}^{n}$ of the map $\mathrm{ev}_{1}(\cdot, t): C^{1} \ni \psi \mapsto \mathrm{ev}_{1}(\psi, t) \in \mathbb{R}^{n}$ at $\phi$ exists and is given by $D \mathrm{ev}_{1}(\cdot, t)(\phi) \widehat{\phi}=$ $\widehat{\phi}(t)=\operatorname{ev}(\widehat{\phi}, t)$. This means that the partial derivative $D_{1} \operatorname{ev}_{1}(\phi, t): C^{1} \rightarrow \mathbb{R}^{n}$ exists and is given by $D_{1} \mathrm{ev}_{1}(\phi, t) \widehat{\phi}=\operatorname{ev}(\widehat{\phi}, t)$. Using this in combination with the continuity of $\mathrm{ev}_{0}$ and of the inclusion map $C^{1} \rightarrow C$, one finds that the map

$$
C^{1} \times(-\infty, 0] \times C^{1} \ni(\phi, t, \widehat{\phi}) \mapsto D_{1} \mathrm{ev}_{1}(\phi, t) \widehat{\phi} \in \mathbb{R}^{n}
$$

is continuous. Next, for $\phi \in C^{1}, t<0, s \in \mathbb{R}$ with $s \neq 0$, and for all $h \neq 0$ with $t+h s<0$,

$$
\frac{1}{h}(\operatorname{ev}(\phi, t+h s)-\operatorname{ev}(\phi, t))=s \frac{\phi(t+s h)-\phi(t)}{s h} \rightarrow s \phi^{\prime}(t)
$$


as $h \rightarrow 0$. It follows that the directional derivative $D \operatorname{ev}_{1}(\phi, \cdot)(t) s$ exists and is given by $D \operatorname{ev}_{1}(\phi, \cdot)(t) s=s \phi^{\prime}(t)=s \operatorname{ev}\left(\phi^{\prime}, t\right)$. The same holds for $s=0$. So the partial derivative $D_{2} \operatorname{ev}_{1}(\phi, t): \mathbb{R} \rightarrow \mathbb{R}^{n}$ exists and is given by $D_{2} \operatorname{ev}_{1}(\phi, t) s=$ $s \operatorname{ev}\left(\phi^{\prime}, t\right)$. Using this in combination with the continuity of $\partial, \mathrm{ev}_{0}$, and of the multiplication on $\mathbb{R}$ one finds that the map

$$
C^{1} \times(-\infty, 0) \times \mathbb{R} \ni(\phi, t, s) \mapsto D_{2} \mathrm{ev}_{1}(\phi, t) s \in \mathbb{R}^{n}
$$

is continuous. Now the assertion follows (see [6]).

Consider the special case $x^{\prime}(t)=a x(t-\delta(x(t)))(\in \mathbb{R})$ of equation (1.2), with $0 \neq a \in \mathbb{R}$ and a continuously differentiable function $\delta: \mathbb{R} \rightarrow[0, \infty)$, which is equation (1.1) with $f: \mathbb{R}^{(-\infty, 0]} \rightarrow \mathbb{R}, f=a \mathrm{ev} \circ(\mathrm{id} \times(-\delta \circ \operatorname{ev}(\cdot, 0)))$. The chain rule in combination with Proposition 2.1 shows that the restriction $f_{1}=\left.f\right|_{C^{1}}$ is continuously differentiable with

$$
\begin{aligned}
D f_{1}(\phi) \widehat{\phi} & =a D \operatorname{ev}_{1}(\phi,-\delta(\phi(0)))\left(\widehat{\phi},-\delta^{\prime}(\phi(0)) \widehat{\phi}(0)\right) \\
& =a\left[\widehat{\phi}(-\delta(\phi(0)))-\delta^{\prime}(\phi(0)) \widehat{\phi}(0) \phi^{\prime}(-\delta(\phi(0)))\right]
\end{aligned}
$$

Moreover, $f_{1}$ has property (e) since the last term does not involve $\widehat{\phi}^{\prime}$ : For $\phi \in C^{1}$ and $\chi \in C$ define

$$
D_{\mathrm{e}} f_{1}(\phi) \chi=a\left[\chi(-\delta(\phi(0)))-\delta^{\prime}(\phi(0)) \chi(0) \phi^{\prime}(-\delta(\phi(0)))\right]
$$

Then the continuity of $\mathrm{ev}_{0}$ and of the inclusion and differentiation maps $C^{1} \rightarrow C$ shows that the map $C^{1} \times C \ni(\phi, \chi) \mapsto D_{\mathrm{e}} f_{1}(\phi) \chi \in \mathbb{R}$ is continuous.

From now on let a continuously differentiable functional $f: C^{1} \supset U \rightarrow \mathbb{R}^{n}$ with property (e) be given. Its solution manifold $X=X_{f}=\left\{\phi \in U: \phi^{\prime}(0)=\right.$ $f(\phi)\}$ is the zeroset of the continuously differentiable map

$$
g: U \ni \phi \mapsto \mathrm{ev}_{0}(\partial \phi, 0)-f(\phi) \in \mathbb{R}^{n} .
$$

The nullspaces $Y=Y_{\phi}=Y_{f, \phi}=\left\{\chi \in C^{1}: \chi^{\prime}(0)=D f(\phi) \chi\right\}=D g(\phi)^{-1}(0)$, for $\phi \in U$, are closed, and for $\phi \in X, T_{\phi} X \subset Y_{\phi}$ because every $\chi \in T_{\phi} X$ equals $\chi=c^{\prime}(0)$ for a continuously differentiable curve $c: I \rightarrow C^{1}$ with $I$ open and $0 \in I, c(0)=\phi, c(I) \subset X, c^{\prime}(0)=\chi$, hence $g \circ c=0$, and the chain rule yields $D g(\phi) \chi=D g(c(0)) D c(0) 1=D(g \circ c) 1=0$, or equivalently, $\chi \in D g(\phi)^{-1}(0)=Y_{\phi}$.

Proposition 2.2. For every $\phi \in U$ the derivative $D g(\phi): C^{1} \rightarrow \mathbb{R}^{n}$ is surjective, and there is a subspace $Q_{\phi} \subset C^{1}$ with $\operatorname{dim} Q_{\phi}=n$ and $C^{1}=Y_{\phi} \oplus Q_{\phi}$.

Proof. 1. Let $\phi \in U$ be given. For surjectivity of the linear map $D g(\phi)$ it is sufficient to show that the range is dense. In order to prove this let $y \in \mathbb{R}^{n}$ and $\varepsilon>0$ be given. As $D_{\mathrm{e}} f(\phi): C \rightarrow \mathbb{R}^{n}$ is continuous (at $0 \in C$ ) there is 
a neighbourhood $N$ of 0 in $C$ with $\left|D_{\mathrm{e}} f(\phi) \chi\right|<\varepsilon$ for all $\chi \in N . N$ contains a neighbourhood of the form

$$
\left\{\psi \in C: \max _{-j \leq t \leq 0}|\psi(t)|<\frac{1}{j}\right\},
$$

with some $j \in \mathbb{N}$, which in turn contains elements $\psi \in C^{1}$ with $\psi^{\prime}(0)=y$, hence

$$
|D g(\phi) \psi-y|=\left|\psi^{\prime}(0)-D f(\phi) \psi-y\right|=\left|D_{\mathrm{e}} f(\phi) \psi\right|<\varepsilon .
$$

2. Choose a basis of $\mathbb{R}^{n}$, take preimages $\phi_{1}, \ldots, \phi_{n}$ under $D g(\phi)$, and consider the span $Q_{\phi}$ of these preimages. Then $\operatorname{dim} Q_{\phi}=n$ and

$$
C^{1}=(D g(\phi))^{-1}(0) \oplus Q_{\phi}=Y_{\phi} \oplus Q_{\phi} .
$$

Recall that finite-dimensional subspaces of a topological vectorspace are closed. The next result implies that $X_{f}$ is a continuously differentiable submanifold of codimension $n$ in the space $C^{1}$.

Proposition 2.3. For every $\phi \in X_{f}$ there are open neighbourhoods $N_{Y}$ of 0 in $Y_{\phi}, N_{Q}$ of 0 in $Q_{\phi}$, and a continuously differentiable map $\xi: N_{Y} \rightarrow Q_{\phi}$ with $\xi\left(N_{Y}\right) \subset N_{Q}$ and $\xi(0)=0$ and

$$
X_{f} \cap\left(N_{Y}+N_{Q}\right)=\left\{\phi+\psi+\xi(\psi) \in C^{1}: \psi \in N_{Y}\right\} .
$$

Proof. Let $\phi \in X=X_{f}=g^{-1}(0)$ be given, write $Y=Y_{\phi}, Q=Q_{\phi}$. The restriction $\left.D g(\phi)\right|_{Q}$ is an isomorphism, due to Proposition 2.2. The map $j: Y \times Q \rightarrow C^{1}$ given by $j(\psi, \chi)=\phi+\psi+\chi$ is affine linear and continuous and thereby continuously differentiable. The set $V=j^{-1}(U)$ is open with $(0,0) \in V$, the map $h=\left.g \circ j\right|_{V}$ is continuously differentiable with $h(0,0)=0$ and

$$
D_{2} h(\psi, \chi) \eta=D h(\psi, \chi)(0, \eta)=D g(j(\psi, \chi)) D j(\psi, \chi)(0, \eta)=D g(j(\psi, \chi)) \eta
$$

for all $(\psi, \chi) \in V$ and all $\eta \in Q$. The equations $D_{2} h(0,0) \eta=D g(\phi) \eta$ for $\eta \in Q$ show that $D_{2} h(0,0): Q \rightarrow \mathbb{R}^{n}$ is an isomorphism. The Implicit Function Theorem 7.3 in combination with the remark preceding it yields open neighbourhoods $N_{Y}$ of 0 in $Y, N_{Q}$ of 0 in $Q$, and a continuously differentiable map $\xi: N_{Y} \rightarrow Q$ with $N_{Y} \times N_{Q} \subset V, \xi\left(N_{Y}\right) \subset N_{Q}, \xi(0)=0$ and

$$
h^{-1}(0) \cap\left(N_{Y} \times N_{Q}\right)=\left\{(\psi, \xi(\psi)) \in Y \times Q: \psi \in N_{Y}\right\} .
$$

Applying $j$, we obtain the assertion.

The idea how to solve the IVP

$$
x^{\prime}(t)=f\left(x_{t}\right) \quad \text { for } t \geq 0, \quad x_{0}=\phi \in X_{f}
$$

is as in [14]. Suppose $x:(-\infty, T] \rightarrow \mathbb{R}^{n}, T>0$, is a solution of equation (1.1) on $[0, T]$ with $x_{0}=\phi$. Continue $\phi$ by $\phi(t)=\phi(0)+t \phi^{\prime}(0)$ to a continuously 
differentiable function $\widehat{\phi}:(-\infty, T] \rightarrow \mathbb{R}^{n}$. Then $y=x-\widehat{\phi}$ satisfies $y(t)=0$ for $t \leq 0$, and for $0 \leq t \leq T$ the equation

$$
\begin{aligned}
y(t) & =x(t)-\widehat{\phi}(t)=x(0)+\int_{0}^{t} f\left(x_{s}\right) d s-\phi(0)-t \phi^{\prime}(0) \\
& =\int_{0}^{t} f\left(y_{s}+\widehat{\phi}_{s}\right) d s-t f(\phi)=\int_{0}^{t}\left(f\left(y_{s}+\widehat{\phi}_{s}\right)-f(\phi)\right) d s
\end{aligned}
$$

holds, with the parameter $\phi \in X_{f} \subset U \subset C^{1}$. Obviously, $y(0)=0=y^{\prime}(0)$. So $\eta=\left.y\right|_{[0, T]} \in C_{0 T, 0}^{1}$ satisfies the fixed point equation

$$
\eta(t)=\int_{0}^{t}\left(f\left(\widehat{\eta}_{s}+\widehat{\phi}_{s}\right)-f(\phi)\right) d s, \quad 0 \leq t \leq T,
$$

where $\widehat{\eta} \in C_{T}^{1}$ is the prolongation of $\eta$ given by $\widehat{\eta}(t)=0$ for all $t<0$. In order to find a solution of the IVP one solves the fixed point equation (2.1) by means of a parametrized contraction on a subset of the Banach space $C_{0 T, 0}^{1}$ with the parameter $\phi \in U \subset C^{1}$. For $\phi \in X_{f}$ the associated fixed point $\eta=\eta_{\phi}$ yields a solution $x=\widehat{\eta}+\widehat{\phi}$ of the IVP.

The next section begins with a framework for studying equation (2.1).

\section{Evaluations, substition operator, and prolongations}

The segment evaluation maps

$$
\begin{aligned}
& E_{T}: C_{T} \times(-\infty, T] \ni(\phi, t) \mapsto \phi_{t} \in C, \\
& E_{T}^{1}: C_{T}^{1} \times(-\infty, T] \ni(\phi, t) \mapsto \phi_{t} \in C^{1}, \\
& E_{T}^{10}: C_{T}^{1} \times(-\infty, T] \ni(\phi, t) \mapsto \phi_{t} \in C
\end{aligned}
$$

for $T \in \mathbb{R}$ and their analogues $E_{\infty}, E_{\infty}^{1}$ for $T=\infty$ are all linear in the first argument.

Proposition 3.1. Let $T \leq \infty$.

(a) The maps $E_{T}$ and $E_{T}^{1}$ are continuous.

(b) For every $\phi \in C_{T}^{1}$ the curve $\Phi:(-\infty, T) \ni t \mapsto \phi_{t} \in C$ is continuously differentiable, with $\Phi^{\prime}(t)=E_{T}\left(\partial_{T} \phi, t\right)$.

(c) The map $\left.E_{T}^{10}\right|_{C_{T}^{1} \times(-\infty, T)}$ is continuously differentiable, with

$$
\begin{aligned}
D_{1} E_{T}^{10}(\phi, t) \widehat{\phi} & =E_{T}^{10}(\widehat{\phi}, t)=\widehat{\phi}_{t} \quad \text { and } \\
D_{2} E_{T}^{10}(\phi, t) s & =s E_{T}^{10}\left(\partial_{T} \phi, t\right)=s\left(\partial_{T} \phi\right)_{t}=s\left(\phi^{\prime}\right)_{t} .
\end{aligned}
$$

Proof. 1. Let $T \in \mathbb{R}$.

1.1. Continuity of $E_{T}$. Let $\phi_{k} \rightarrow \phi$ in $C_{T}$ as $\mathbb{N} \ni k \rightarrow \infty$, and $t_{k} \rightarrow t$ in $(-\infty, T]$. Let $j \in \mathbb{N}$ be given. In order to show $\left|\phi_{k, t_{k}}-\phi_{t}\right|_{j} \rightarrow 0$ as $k \rightarrow \infty$, 
choose $m>T$ in $\mathbb{N}$ with $T-m<t$. There exists $k_{m} \in \mathbb{N}$ such that for all integers $k \geq k_{m}, T-m \leq t_{k} \leq T$. For such $k$,

$$
\begin{aligned}
\left|\phi_{k, t_{k}}-\phi_{t}\right|_{j} & =\max _{-j \leq s \leq 0}\left|\phi_{k}\left(t_{k}+s\right)-\phi(t+s)\right| \\
& \leq \max _{-j \leq s \leq 0}\left|\phi_{k}\left(t_{k}+s\right)-\phi\left(t_{k}+s\right)\right|+\max _{-j \leq s \leq 0}\left|\phi\left(t_{k}+s\right)-\phi(t+s)\right| \\
& \leq \max _{T-m-j \leq u \leq T}\left|\phi_{k}(u)-\phi(u)\right|+\max _{-j \leq s \leq 0}\left|\phi\left(t_{k}+s\right)-\phi(t+s)\right| \\
& =\left|\phi_{k}-\phi\right|_{T, m+j}+\max _{-j \leq s \leq 0}\left|\phi\left(t_{k}+s\right)-\phi(t+s)\right| .
\end{aligned}
$$

Use $\left|\phi_{k}-\phi\right|_{T, m+j} \rightarrow 0$ as $k \rightarrow \infty$ and the uniform continuity of $\phi$ on the compact interval $[T-m-j, T]$ in order to complete the proof.

1.2. Continuity of $E_{T}^{1}$. For $j \in \mathbb{N}$ and $\phi, \psi$ in $C_{T}^{1}$ and $t, s \in(-\infty, T]$,

$$
\begin{aligned}
\left|\phi_{t}-\psi_{s}\right|_{1, j} & =\left|\phi_{t}-\psi_{s}\right|_{j}+\left|\left(\phi_{t}\right)^{\prime}-\left(\psi_{s}\right)^{\prime}\right|_{j} \\
& =\left|E_{T}(\phi, t)-E_{T}(\psi, s)\right|_{j}+\left|\left(\phi^{\prime}\right)_{t}-\left(\psi^{\prime}\right)_{s}\right|_{j} \\
& =\left|E_{T}(\phi, t)-E_{T}(\psi, s)\right|_{j}+\left|E_{T}\left(\partial_{T} \phi, t\right)-E_{T}\left(\partial_{T} \psi, s\right)\right|_{j} .
\end{aligned}
$$

Use the continuity of $E_{T}$ and $\partial_{T}$ in order to complete the proof.

1.3. As the inclusion $C^{1} \rightarrow C$ is continuous, it follows that $E_{T}^{10}$ is continuous.

1.4. On the map $\Phi$, for $\phi \in C_{T}^{1}$. Let $t<T, j \in \mathbb{N}, 0 \neq h \in \mathbb{R}, t+h<T$. Then

$$
\begin{aligned}
\left|\frac{1}{h}(\Phi(t+h)-\Phi(t))-E_{T}\left(\partial_{T} \phi, t\right)\right|_{j} \\
\quad=\max _{-j \leq s \leq 0}\left|\frac{1}{h}(\phi(t+h+s)-\phi(t+s))-\phi^{\prime}(t+s)\right| \\
\quad=\max _{-j \leq s \leq 0}\left|\int_{0}^{1}\left(\phi^{\prime}(t+s+\theta[t+h+s-(t+s)])-\phi^{\prime}(t+s)\right) d \theta\right| \\
\quad \leq \max _{-j \leq s \leq 0} \int_{0}^{1}\left|\phi^{\prime}(t+s+\theta h)-\phi^{\prime}(t+s)\right| d \theta,
\end{aligned}
$$

and the uniform continuity of $\phi^{\prime}$ on $[t-1-j, T]$ implies that the last term tends to 0 as $0 \neq h \rightarrow 0$. This shows that $\Phi$ is differentiable with $\Phi^{\prime}(t)=E_{T}\left(\partial_{T} \phi, t\right)$. Using (a), one finds that $\Phi^{\prime}$ is continuous.

1.5. On $\left.E_{T}^{10}\right|_{C_{T}^{1} \times(-\infty, T)}$. Let $\phi \in C_{T}^{1}$ and $t<T$. The existence of $D_{1} E_{T}^{10}(\phi, t)$ and the formula $D_{1} E_{T}^{10}(\phi, t) \widehat{\phi}=E_{T}^{10}(\widehat{\phi}, t)=\widehat{\phi}_{t}$ follow from linearity in the first argument. Using part 1.3 of the proof, one concludes that the map

$$
C_{T}^{1} \times(-\infty, T) \times C_{T}^{1} \ni(\phi, t, \widehat{\phi}) \mapsto D_{1} E_{T}^{10}(\phi, t) \widehat{\phi} \in C
$$

is continuous.

The existence of $D_{2} E_{T}^{10}(\phi, t) s$ for $s \in \mathbb{R}$ follows from $E_{T}^{10}(\phi, t+h s)=\Phi(t+h s)$ for $t+h s<T$ in combination with (b) and the chain rule. Also, $D_{2} E_{T}^{10}(\phi, t) s=$ 
$s \Phi^{\prime}(t)=s E_{T}^{10}\left(\partial_{T} \phi, t\right)$, which in combination with part 1.3 shows that the map

$$
C_{T}^{1} \times(-\infty, T) \times \mathbb{R} \ni(\phi, t, s) \mapsto D_{2} E_{T}^{10}(\phi, t) s \in C
$$

is continuous. Now it follows that $E_{T}^{10}$ is continuously differentiable (see [6]).

2. The proofs for $T=\infty$ are analogous.

Next, consider the substitution operator $F_{T}: \operatorname{dom}_{T} \rightarrow C_{0 T}$ which, for $0<$ $T<\infty$, is given by

$$
\operatorname{dom}_{T}=\left\{\phi \in C_{T}^{1}: \phi_{s} \in U \text { for } 0 \leq s \leq T\right\}
$$

and

$$
F_{T}(\phi)(t)=f\left(\phi_{t}\right)=f\left(E_{T}^{1}(\phi, t)\right) \in \mathbb{R}^{n} .
$$

Notice that in order to obtain continuous differentiability of $F_{T}$ the chain rule cannot be applied, due to lack of smoothness of the map $E_{T}^{1}$.

Proposition 3.2. Let $0<T<\infty$. The set $\operatorname{dom}_{T}$ is open and $F_{T}$ is continuously differentiable with

$$
\left(D F_{T}(\phi) \widehat{\phi}\right)(s)=D_{\mathrm{e}} f\left(E_{T}^{1}(\phi, s)\right) E_{T}^{10}(\widehat{\phi}, s) .
$$

Proof. 1. (Openness) Let $\phi \in \operatorname{dom}_{T}$. Due to the continuity of $E_{T}^{1}$ for each $t \in[0, T]$ there are open neighbourhoods $N_{t}$ of $\phi$ in $C_{T}^{1}$ and $V_{t}$ of $t$ in $\mathbb{R}$ with $\psi_{s}=E_{T}^{1}(\psi, s) \in U$ for all $\psi \in N_{t}, s \in V_{t} \cap[0, T]$. Due to compactness there exists a finite subset $\tau \subset[0, T]$ with $[0, T] \subset \bigcup_{t \in \tau} V_{t}$. Then $\bigcap_{t \in \tau} N_{t}$ is a neighbourhood of $\phi$ in $\operatorname{dom}_{T}$.

2. For every $\phi \in \operatorname{dom}_{T}$ and every $\widehat{\phi} \in C_{T}^{1}$ the map

$$
[0, T] \ni t \mapsto D_{\mathrm{e}} f\left(E_{T}^{1}(\phi, t)\right) E_{T}^{10}(\widehat{\phi}, t) \in \mathbb{R}^{n}
$$

is continuous. Therefore, the equation

$$
B_{T}(\phi, \widehat{\phi})(t)=D_{\mathrm{e}} f\left(E_{T}^{1}(\phi, t)\right) E_{T}^{10}(\widehat{\phi}, t)
$$

defines a map $B_{T}$ : $\operatorname{dom}_{T} \times C_{T}^{1} \rightarrow C_{0 T}$. In order to show that $B_{T}$ is continuous, assume $\phi_{k} \rightarrow \phi$ in $\operatorname{dom}_{T}$ and $\widehat{\phi}_{k} \rightarrow \widehat{\phi}$ in $C_{T}^{1}$ as $\mathbb{N} \ni k \rightarrow \infty$. For all $k \in \mathbb{N}$,

$$
\begin{aligned}
\mid B_{T}\left(\phi_{k}, \widehat{\phi}_{k}\right)- & \left.B_{T}(\phi, \widehat{\phi})\right|_{0 T} \\
& =\max _{0 \leq t \leq T}\left|D_{\mathrm{e}} f\left(E_{T}^{1}\left(\phi_{k}, t\right)\right) E_{T}^{10}\left(\widehat{\phi}_{k}, t\right)-D_{\mathrm{e}} f\left(E_{T}^{1}(\phi, t)\right) E_{T}^{10}(\widehat{\phi}, t)\right| .
\end{aligned}
$$

The map $\operatorname{dom}_{T} \times C_{T}^{1} \times[0, T] \ni(\chi, \widehat{\chi}, t) \mapsto D_{\mathrm{e}} f\left(E_{T}^{1}(\chi, t)\right) E_{T}^{10}(\widehat{\chi}, t) \in \mathbb{R}^{n}$ is uniformly continuous on the compact set $\{(\phi, \widehat{\phi})\} \times[0, T]$.

Let $\varepsilon>0$. Then there is a neighbourhood $N$ of $(\phi, \widehat{\phi})$ in $\operatorname{dom}_{T} \times C_{T}^{1}$ such that for all $(\psi, \widehat{\psi}) \in N$ and for all $t \in[0, T]$,

$$
\begin{aligned}
\left|B_{T}(\psi, \widehat{\psi})(t)-B_{T}(\phi, \widehat{\phi})(t)\right| & \\
= & \left|D_{\mathrm{e}} f\left(E_{T}^{1}(\psi, t)\right) E_{T}^{10}(\widehat{\psi}, t)-D_{\mathrm{e}} f\left(E_{T}^{1}(\phi, t)\right) E_{T}^{10}(\widehat{\phi}, t)\right|<\varepsilon .
\end{aligned}
$$


There exists an integer $k_{N}$ with $\left(\phi_{k}, \widehat{\phi}_{k}\right) \in N$ for all integers $k \geq k_{N}$. For such $k$,

$$
\left|B_{T}\left(\phi_{k}, \widehat{\phi}_{k}\right)-B_{T}(\phi, \widehat{\phi})\right|_{0 T}<\varepsilon \text {. }
$$

3. (Directional derivatives) Let $\phi \in \operatorname{dom}_{T}, \widehat{\phi} \in C_{T}^{1}$ be given. There is a convex neighbourhood $N$ of $\phi$ in $\operatorname{dom}_{T}$, and there exists $\eta>0$ with $\phi+h \widehat{\phi} \in N$ for all $h \in(-\eta, \eta)$. Hence $\phi+[0,1] h \widehat{\phi} \subset N$ for $|h|<\eta$, and thereby

$$
\phi_{t}+\theta h \widehat{\phi}_{t}=(\phi+\theta h \widehat{\phi})_{t} \in U \text { for }|h|<\eta, 0 \leq \theta \leq 1,0 \leq t \leq T .
$$

For $0<|h|<\eta$ it follows that

$$
\begin{aligned}
& \left|\frac{1}{h}\left(F_{T}(\phi+h \widehat{\phi})-F_{T}(\phi)\right)-B_{T}(\phi, \widehat{\phi})\right|_{0 T} \\
& =\max _{0 \leq t \leq T}\left|\frac{1}{h}\left(f\left((\phi+h \widehat{\phi})_{t}\right)-f\left(\phi_{t}\right)\right)-D_{\mathrm{e}} f\left(E_{T}^{1}(\phi, t)\right) E_{T}^{10}(\widehat{\phi}, t)\right| \\
& =\max _{0 \leq t \leq T}\left|\frac{1}{h} \int_{0}^{1}\left(D f\left(\phi_{t}+\theta h \widehat{\phi}_{t}\right) h \widehat{\phi}_{t}-D_{\mathrm{e}} f\left(E_{T}^{1}(\phi, t)\right) E_{T}^{10}(\widehat{\phi}, t)\right) d \theta\right| \\
& =\max _{0 \leq t \leq T}\left|\int_{0}^{1}\left(D_{\mathrm{e}} f\left(E_{T}^{1}(\phi+\theta h \widehat{\phi}, t)\right) E_{T}^{10}(\widehat{\phi}, t)-D_{\mathrm{e}} f\left(E_{T}^{1}(\phi, t)\right) E_{T}^{10}(\widehat{\phi}, t)\right) d \theta\right| .
\end{aligned}
$$

The map

$$
[0, T] \times(-\eta, \eta) \times[0,1] \ni(t, h, \theta) \mapsto D_{\mathrm{e}} f\left(E_{T}^{1}(\phi+\theta h \widehat{\phi}, t)\right) E_{T}^{10}(\widehat{\phi}, t) \in \mathbb{R}^{n}
$$

is continuous, hence uniformly continuous on the compact set $[0, T] \times\{0\} \times[0,1]$. Let $\varepsilon>0$. Then there exists $\delta_{\varepsilon} \in(0, \eta)$ such that for all $t \in[0, T], h \in\left(-\delta_{\varepsilon}, \delta_{\varepsilon}\right)$, $\theta \in[0,1]$,

$$
\begin{aligned}
& \left|D_{\mathrm{e}} f\left(E_{T}^{1}(\phi+\theta h \widehat{\phi}, t)\right) E_{T}^{10}(\widehat{\phi}, t)-D_{\mathrm{e}} f\left(E_{T}^{1}(\phi, t)\right) E_{T}^{10}(\widehat{\phi}, t)\right| \\
& \quad=\left|D_{\mathrm{e}} f\left(E_{T}^{1}(\phi+\theta h \widehat{\phi}, t)\right) E_{T}^{10}(\widehat{\phi}, t)-D_{\mathrm{e}} f\left(E_{T}^{1}(\phi+\theta \cdot 0 \cdot \widehat{\phi}, t)\right) E_{T}^{10}(\widehat{\phi}, t)\right|<\varepsilon .
\end{aligned}
$$

It follows that for $0<|h|<\delta_{\varepsilon}$,

$$
\left|\frac{1}{h}\left(F_{T}(\phi+h \widehat{\phi})-F_{T}(\phi)\right)-B_{T}(\phi, \widehat{\phi})\right|_{0 T}<\varepsilon .
$$

Therefore $D F_{T}(\phi) \widehat{\phi}$ exists and is equal to $B_{T}(\phi, \widehat{\phi})$. Using part 2, one finds that $F_{T}$ is continuously differentiable.

The prolongation maps $P_{T}: C^{1} \rightarrow C_{T}^{1}, 0<T \leq \infty$, given by

$$
P_{T} \phi(t)=\phi(t) \quad \text { for } t \leq 0, \quad P_{T} \phi(t)=\phi(0)+t \phi^{\prime}(0) \quad \text { for } 0<t \leq T,
$$

$P_{S T}: C_{0 S}^{1} \rightarrow C_{0 T}^{1}, 0<S<T<\infty$, given by

$$
\begin{array}{ll}
P_{S T} \phi(t)=\phi(t) & \text { for } 0 \leq t \leq S, \\
P_{S T} \phi(t)=\phi(S)+(t-S) \phi^{\prime}(S) & \text { for } S<t \leq T,
\end{array}
$$


$Z_{T}: C_{0 T, 0} \rightarrow C_{T}, 0<T<\infty$, given by

$$
Z_{T} \phi(t)=\phi(t) \quad \text { for } 0 \leq t \leq T, \quad Z_{T}(\phi)(t)=0 \quad \text { for } t<0,
$$

and the integration operators $I_{T}: C_{0 T, 0} \rightarrow C_{0 T, 0}^{1}, 0<T<\infty$, given by

$$
I_{T} \phi(t)=\int_{0}^{t} \phi(s) d s
$$

are all linear and continuous. Obviously, $Z_{T} C_{0 T, 0}^{1} \subset C_{T}^{1}$. For $P_{S T}, 0<S<T$, $P_{S T} C_{0 S, 0}^{1} \subset C_{0 T, 0}^{1}$, and

$$
\left|P_{S T} \phi\right|_{1,0 T} \leq(2+T)|\phi|_{1,0 S} \quad \text { for all } \phi \in C_{0 S}^{1}
$$

because of the estimate

$$
\begin{aligned}
\left|P_{S T} \phi\right|_{1,0 T} & =\max _{0 \leq t \leq T}\left|P_{S T} \phi(t)\right|+\max _{0 \leq t \leq T}\left|\left(P_{S T} \phi\right)^{\prime}(t)\right| \\
& \leq \max _{0 \leq t \leq S}|\phi(t)|+|\phi(S)|+\left|\phi^{\prime}(S)\right| T+\max _{0 \leq t \leq S}\left|\phi^{\prime}(t)\right| .
\end{aligned}
$$

It follows that, for every $T>0$, the set

$$
D_{T}=\left\{(\phi, \eta) \in U \times C_{0 T, 0}^{1}: P_{T} \phi+Z_{T} \eta \in \operatorname{dom}_{T}\right\}
$$

is open, and the map

$$
G_{T}: D_{T} \rightarrow C_{0 T, 0}, \quad G_{T}(\phi, \eta)(t)=F_{T}\left(P_{T} \phi+Z_{T} \eta\right)(t)-f(\phi) \in \mathbb{R}^{n},
$$

$\left(\right.$ with $\left.G_{T}(\phi, \eta)(0)=f\left(\left(P_{T} \phi+Z_{T} \eta\right)_{0}\right)-f(\phi)=f(\phi+0)-f(\phi)=0\right)$ is continuously differentiable, because of the chain rule, continuity of the linear maps $P_{T}$ and $Z_{T}$, Proposition 3.2, the continuous differentiability of $f$, the continuity of the linear mapping $\tau: \mathbb{R}^{n} \rightarrow C_{0 T}$ given by $\tau(\xi)(t)=\xi$, and the fact that the vectorspace operations of $C_{0 T}$ and $C_{T}^{1}$ are continuous.

Corollary 3.3. Let $0<T<\infty$. For $(\phi, \eta) \in D_{T}$ and $\widehat{\phi} \in C^{1}, \widehat{\eta} \in C_{0 T, 0}^{1}$,

$$
D G_{T}(\phi, \eta)(\widehat{\phi}, \widehat{\eta})=D F_{T}\left(P_{T} \phi+Z_{T} \eta\right)\left(P_{T} \widehat{\phi}+Z_{T} \widehat{\eta}\right)-\tau(D f(\phi) \widehat{\phi}),
$$

and, for $0 \leq t \leq T$,

$$
\begin{aligned}
D G_{T}(\phi, \eta) & (\widehat{\phi}, \widehat{\eta})(t) \\
& =D_{\mathrm{e}} f\left(E_{T}^{1}\left(P_{T} \phi+Z_{T} \eta, t\right)\right) E_{T}^{10}\left(P_{T} \widehat{\phi}+Z_{T} \widehat{\eta}, t\right)-\tau(D f(\phi) \widehat{\phi})(t) \\
& =D_{\mathrm{e}} f\left(\left(P_{T} \phi\right)_{t}+\left(Z_{T} \eta\right)_{t}\right)\left(\left(P_{T} \widehat{\phi}\right)_{t}+\left(Z_{T} \widehat{\eta}\right)_{t}\right)-D f(\phi) \widehat{\phi} .
\end{aligned}
$$

The map $A_{T}=I_{T} \circ G_{T}$ is continuously differentiable.

The next result prepares the proof that $A_{T}$ with $T>0$ sufficiently small defines a uniform contraction on a small ball in $C_{0 T, 0}^{1}$. 
Proposition 3.4. Let $\phi \in U$ be given. There exist $T=T_{\phi}>0$, a neighbourhood $V=V_{\phi}$ of $\phi$ in $U, \varepsilon=\varepsilon_{\phi}>0$, and $j=j_{\phi} \in \mathbb{N}$ such that for all $S \in(0, T), \chi \in V, \eta$ and $\widetilde{\eta}$ in $C_{0 S, 0}^{1}$ with $|\eta|_{1,0 S}<\varepsilon$ and $|\widetilde{\eta}|_{1,0 S}<\varepsilon, w \in[0, S]$, and $\theta \in[0,1]$,

$$
\left(P_{S} \chi\right)_{w}+\left(Z_{S} \eta\right)_{w}+\theta\left[\left(Z_{S} \widetilde{\eta}\right)_{w}-\left(Z_{S} \eta\right)_{w}\right] \in U
$$

and

$$
\begin{array}{r}
\left|D_{\mathrm{e}} f\left(\left(P_{S} \chi\right)_{w}+\left(Z_{S} \eta\right)_{w}+\theta\left[\left(Z_{S} \widetilde{\eta}\right)_{w}-\left(Z_{S} \eta\right)_{w}\right]\right)\left[\left(Z_{S} \widetilde{\eta}\right)_{w}-\left(Z_{S} \eta\right)_{w}\right]\right| \\
\leq 2 j|\widetilde{\eta}-\eta|_{0 S} .
\end{array}
$$

Proof. 1. Let $\phi \in U$ be given. As the map $U \times C \ni(\chi, \eta) \mapsto D_{\mathrm{e}} f(\chi) \eta \in \mathbb{R}^{n}$ is continuous at $(\phi, 0)$, there are neighbourhoods $V^{\prime}$ of $\phi$ in $U$ and $N$ of 0 in $C$ with

$$
\left|D_{\mathrm{e}} f(\chi) \eta\right|=\left|D_{\mathrm{e}} f(\chi) \eta-D_{\mathrm{e}} f(\phi) 0\right|<1 \quad \text { for all } \chi \in V^{\prime}, \eta \in N \text {. }
$$

There exists $j=j_{N} \in \mathbb{N}$ with $\left\{\zeta \in C:|\zeta|_{j}<1 / j\right\} \subset N$.

2. By the continuity of the map $\mathbb{R} \ni t \mapsto E_{\infty}^{1}\left(P_{\infty} \phi, t\right) \in C^{1}$ at $t=0$, with $E_{\infty}^{1}\left(P_{\infty} \phi, 0\right)=\phi$, there exists $T>0$ with $E_{\infty}^{1}\left(P_{\infty} \phi, t\right) \in V^{\prime}$ for all $t \in[0, T]$. The continuous map

$$
\alpha: C^{1} \times C_{0 T, 0}^{1} \times[0, T] \ni(\chi, \eta, t) \mapsto E_{\infty}^{1}\left(P_{\infty} \chi, t\right)+E_{T}^{1}\left(Z_{T} \eta, t\right) \in C^{1}
$$

satisfies $\alpha(\phi, 0, t)=E_{\infty}^{1}\left(P_{\infty} \phi, t\right) \in V^{\prime}$ for all $t \in[0, T]$ and is uniformly continuous on the compact set $\{\phi\} \times\{0\} \times[0, T]$. It follows that there exist a neighbourhood $V$ of $\phi$ in $V^{\prime}$ and $\varepsilon^{\prime}>0$ such that

$$
E_{\infty}^{1}\left(P_{\infty} \chi, t\right)+E_{T}^{1}\left(Z_{T} \eta, t\right)=\alpha(\chi, \eta, t) \in V^{\prime}
$$

for all $\chi \in V, \eta \in C_{0 T, 0}^{1}$ with $|\eta|_{1,0 T}<\varepsilon^{\prime}$, and $t \in[0, T]$. Observe that $E_{\infty}^{1}\left(P_{\infty} \chi, t\right)=E_{T}^{1}\left(P_{T} \chi, t\right)$ for these $\chi$ and $t$.

3. Set $\varepsilon=\varepsilon^{\prime} /(2+T)$. Let $0<S<T$ and let $\chi \in V, \eta \neq \widetilde{\eta}$ in $C_{0 S, 0}^{1}$ be given, with $|\eta|_{1,0 S}<\varepsilon$ and $|\widetilde{\eta}|_{1,0 S}<\varepsilon$. Let $0 \leq w \leq S, 0 \leq \theta \leq 1$. Then

$$
\left|P_{S T} \eta\right|_{1,0 T} \leq(2+T)|\eta|_{1,0 S}<\varepsilon^{\prime}
$$

(see (3.1)) and analogously $\left|P_{S T} \widetilde{\eta}\right|_{1,0 T}<\varepsilon^{\prime}$. By convexity,

$$
\left|P_{S T} \eta+\theta\left[P_{S T} \tilde{\eta}-P_{S T} \eta\right]\right|_{1,0 T}<\varepsilon^{\prime} .
$$

The choice of $V$ and $\varepsilon^{\prime}$ in part 2 yields

$$
V^{\prime} \ni E_{\infty}^{1}\left(P_{\infty} \chi, w\right)+E_{T}^{1}\left(Z_{T}\left(P_{S T} \eta+\theta\left[P_{S T} \widetilde{\eta}-P_{S T} \eta\right]\right), w\right) .
$$

Due to $0 \leq w \leq S$,

$$
E_{T}^{1}\left(Z_{T} P_{S T} \eta, w\right)=\left(Z_{S} \eta\right)_{w}, \quad E_{T}^{1}\left(Z_{T} P_{S T} \tilde{\eta}, w\right)=\left(Z_{S} \widetilde{\eta}\right)_{w},
$$


and

$$
\begin{aligned}
E_{T}^{1}\left(Z _ { T } \left(P_{S T} \eta\right.\right. & \left.\left.+\theta\left[P_{S T} \widetilde{\eta}-P_{S T} \eta\right]\right), w\right) \\
= & E_{T}^{1}\left(Z_{T} P_{S T} \eta, w\right)+\theta\left[E_{T}^{1}\left(Z_{T} P_{S T} \widetilde{\eta}, w\right)-E_{T}^{1}\left(Z_{T} P_{S T} \eta, w\right)\right] \\
= & \left(Z_{S} \eta\right)_{w}+\theta\left[\left(Z_{S} \widetilde{\eta}\right)_{w}-\left(Z_{S} \eta\right)_{w}\right] .
\end{aligned}
$$

Using this and $E_{\infty}^{1}\left(P_{\infty} \chi, w\right)=\left(P_{S} \chi\right)_{w}$, one arrives at

$$
V^{\prime} \ni\left(P_{S} \chi\right)_{w}+\left(Z_{S} \eta\right)_{w}+\theta\left[\left(Z_{S} \widetilde{\eta}\right)_{w}-\left(Z_{S} \eta\right)_{w}\right] .
$$

4. Set $\zeta=(\widetilde{\eta}-\eta) /\left(2 j|\eta-\widetilde{\eta}|_{0 S}\right) \in C_{0 S}$. Then

$$
\begin{aligned}
\left|\left(Z_{S} \zeta\right)_{w}\right|_{j} & =\max _{-j \leq t \leq 0}\left|\left(Z_{S} \zeta\right)(w+t)\right|=\max _{w-j \leq s \leq w}\left|\left(Z_{S} \zeta\right)(s)\right| \\
& \leq \max _{0 \leq s \leq S}\left|\left(Z_{S} \zeta\right)(s)\right|=\max _{0 \leq s \leq S}|\zeta(s)|=|\zeta|_{0 S}<\frac{1}{j},
\end{aligned}
$$

hence $\left(Z_{S} \zeta\right)_{w} \in N$, and therefore

$$
\begin{aligned}
1> & \left|D_{\mathrm{e}} f\left(\left(P_{S} \chi\right)_{w}+\left(Z_{S} \eta\right)_{w}+\theta\left[\left(Z_{S} \widetilde{\eta}\right)_{w}-\left(Z_{S} \eta\right)_{w}\right]\right)\left(Z_{S} \zeta\right)_{w}\right| \\
= & \mid D_{\mathrm{e}} f\left(\left(P_{S} \chi\right)_{w}+\left(Z_{S} \eta\right)_{w}+\theta\left[\left(Z_{S} \widetilde{\eta}\right)_{w}-\left(Z_{S} \eta\right)_{w}\right]\right) \\
& \times \frac{1}{2 j|\eta-\widetilde{\eta}|_{0 S}}\left(Z_{S}(\widetilde{\eta}-\eta)\right)_{w} \mid \\
= & \mid D_{\mathrm{e}} f\left(\left(P_{S} \chi\right)_{w}+\left(Z_{S} \eta\right)_{w}+\theta\left[\left(Z_{S} \widetilde{\eta}\right)_{w}-\left(Z_{S} \eta\right)_{w}\right]\right) \\
& \times \frac{1}{2 j|\eta-\widetilde{\eta}|_{0 S}}\left(\left(Z_{S} \widetilde{\eta}\right)_{w}-\left(Z_{S} \eta\right)_{w}\right) \mid
\end{aligned}
$$

which implies the assertion.

\section{A parametrized contraction and local solutions}

Let $\phi \in U$, and let $T=T_{\phi}>0$, a convex neighbourhood $V=V_{\phi}$ of $\phi$ in $U$, $\varepsilon=\varepsilon_{\phi}>0$, and $j=j_{\phi} \in \mathbb{N}$ be given as in Proposition 3.4.

Proposition 4.1. For every $S \in(0, T), \chi \in V, \eta$ and $\tilde{\eta}$ in $C_{0 S, 0}^{1}$ with $|\eta|_{1,0 S}<\varepsilon$ and $|\widetilde{\eta}|_{1,0 S}<\varepsilon$,

$(\chi, \eta) \in D_{S},(\chi, \widetilde{\eta}) \in D_{S} \quad$ and $\quad\left|A_{S}(\chi, \widetilde{\eta})-A_{S}(\chi, \eta)\right|_{1,0 S} \leq 2 j S(S+1)|\widetilde{\eta}-\eta|_{1,0 S}$.

Proof. Let $S \in(0, T), \chi \in V, \eta$ and $\widetilde{\eta}$ in $C_{0 S, 0}^{1}$ with $|\eta|_{1,0 S}<\varepsilon$ and $|\widetilde{\eta}|_{1,0 S}<\varepsilon$ be given. Relation (3.2) with $\theta=0$ and $\theta=1$ yields $(\chi, \eta) \in D_{S}$ and $(\chi, \widetilde{\eta}) \in D_{S}$. Moreover,

$$
\begin{aligned}
& \left|A_{S}(\chi, \widetilde{\eta})-A_{S}(\chi, \eta)\right|_{1,0 S}=\left|I_{S}\left(G_{S}(\chi, \widetilde{\eta})-G_{S}(\chi, \eta)\right)\right|_{1,0 S} \\
& \quad \leq S \max _{0 \leq w \leq S}\left|G_{S}(\chi, \widetilde{\eta})(w)-G_{S}(\chi, \eta)(w)\right|+\max _{0 \leq w \leq S}\left|G_{S}(\chi, \widetilde{\eta})(w)-G_{S}(\chi, \eta)(w)\right| \\
& \quad=(S+1) \max _{0 \leq w \leq S}\left|G_{S}(\chi, \widetilde{\eta})(w)-G_{S}(\chi, \eta)(w)\right|
\end{aligned}
$$


and, for every $w \in[0, S]$,

$\left|G_{S}(\chi, \widetilde{\eta})(w)-G_{S}(\chi, \eta)(w)\right| \leq\left|G_{S}(\chi, \widetilde{\eta})-G_{S}(\chi, \eta)\right|_{0 S}$

(smoothness of $G_{S}$, convexity of $V \times\left\{\widehat{\eta} \in C_{0 S, 0}^{1}:|\widehat{\eta}|_{1,0 S}<\varepsilon\right\}$ )

$$
=\left|\int_{0}^{1} D G_{S}(\chi, \eta+\theta[\widetilde{\eta}-\eta])(0, \widetilde{\eta}-\eta) d \theta\right|_{0 S}
$$

$$
\leq \max _{0 \leq \theta \leq 1}\left|D G_{S}(\chi, \eta+\theta[\widetilde{\eta}-\eta])(0, \widetilde{\eta}-\eta)\right|_{0 S} .
$$

The last term equals

$$
\max _{0 \leq \theta \leq 1} \max _{0 \leq w \leq S}\left|D_{\mathrm{e}} f\left(\left(P_{S} \chi\right)_{w}+\left(Z_{S}(\eta+\theta[\widetilde{\eta}-\eta])\right)_{w}\right)\left(Z_{S}(\widetilde{\eta}-\eta)\right)_{w}\right|
$$

(see Corollary 3.3 with $\widehat{\phi}=0$ )

$$
\begin{array}{r}
=\max _{0 \leq \theta \leq 1} \max _{0 \leq w \leq S}\left|D_{\mathrm{e}} f\left(\left(P_{S} \chi\right)_{w}+\left(Z_{S} \eta\right)_{w}+\theta\left[\left(Z_{S} \widetilde{\eta}\right)_{w}-\left(Z_{S} \eta\right)_{w}\right]\right)\left[\left(Z_{S} \widetilde{\eta}\right)_{w}-\left(Z_{S} \eta\right)_{w}\right]\right| \\
\leq 2 j|\widetilde{\eta}-\eta|_{0 S}
\end{array}
$$

(by Proposition 3.4). It follows that

$$
\begin{aligned}
\mid A_{S}(\chi, \widetilde{\eta}) & -\left.A_{S}(\chi, \eta)\right|_{1,0 S} \\
& \leq 2 j(S+1)|\widetilde{\eta}-\eta|_{0 S}=2 j(S+1) \max _{0 \leq w \leq S}\left|\int_{0}^{w}\left(\widetilde{\eta}^{\prime}(s)-\eta^{\prime}(s)\right) d s\right| \\
& \leq 2 j(S+1) S \max _{0 \leq w \leq S}\left|\widetilde{\eta}^{\prime}(s)-\eta^{\prime}(s)\right| \leq 2 j S(2+S)|\widetilde{\eta}-\eta|_{1,0 S} .
\end{aligned}
$$

Proposition 4.2. $\lim _{S \searrow 0} A_{S}(\phi, 0)=0$.

Proof. For $0<S<T$,

$$
\begin{aligned}
\left|A_{S}(\phi, 0)\right|_{1,0 S} & =\left|I_{S} G_{S}(\phi, 0)\right|_{1,0 S} \leq S\left|G_{S}(\phi, 0)\right|_{0 S}+\left|G_{S}(\phi, 0)\right|_{0 S} \\
& =(S+1)\left|G_{S}(\phi, 0)\right|_{0 S}=(S+1) \max _{0 \leq w \leq S}\left|f\left(\left(P_{S} \phi\right)_{w}+0\right)-f(\phi)\right| \\
& =(S+1) \max _{0 \leq w \leq S}\left|f\left(\left(P_{T} \phi\right)_{w}\right)-f\left(\left(P_{T} \phi\right)_{0}\right)\right| \\
& =(S+1) \max _{0 \leq w \leq S}\left|f\left(E_{T}^{1}\left(P_{T} \phi, w\right)\right)-f\left(E_{T}^{1}\left(P_{T} \phi, 0\right)\right)\right|,
\end{aligned}
$$

so continuity of $f, P_{T}$ and $E_{T}^{1}$ yields the assertion.

Proposition 4.3. There exist $S_{\phi} \in\left(0, T_{\phi}\right)$ and an open neighbourhood $W_{\phi}$ of $\phi$ in $V_{\phi}$ such that for all $\chi \in W_{\phi}$, for all $S \in\left(0, S_{\phi}\right]$, and all $\eta \in C_{0 S, 0}^{1}$, $\widetilde{\eta} \in C_{0 S, 0}^{1}$ with $|\eta|_{1,0 S} \leq \varepsilon_{\phi} / 2,|\widetilde{\eta}|_{1,0 S} \leq \varepsilon_{\phi} / 2$,

$$
\begin{gathered}
(\chi, \eta) \in D_{S}, \quad(\chi, \widetilde{\eta}) \in D_{S}, \\
\left|A_{S}(\chi, \eta)\right|_{1,0 S}<\frac{\varepsilon_{\phi}}{2} \quad \text { and } \quad\left|A_{S}(\chi, \widetilde{\eta})-A_{S}(\chi, \eta)\right|_{1,0 S} \leq \frac{1}{2}|\widetilde{\eta}-\eta|_{1,0 S} .
\end{gathered}
$$


Proof. 1. Choose $S_{\phi} \in\left(0, T_{\phi}\right)$ with $\left|A_{S}(\phi, 0)\right|_{1,0 S}<\varepsilon_{\phi} / 6$ for all $S \in\left(0, S_{\phi}\right]$, which is possible due to Proposition 4.2, and

$$
2 j S_{\phi}\left(1+S_{\phi}\right)<\min \left\{\frac{1}{2}, \frac{\varepsilon_{\phi}}{3}\right\} .
$$

As $A_{S_{\phi}}$ is continuous, there exists an open neigbourhood $W_{\phi}$ of $\phi$ in $V_{\phi}$ so that for all $\chi \in W_{\phi}$,

$$
\left|A_{S_{\phi}}(\chi, 0)-A_{S_{\phi}}(\phi, 0)\right|_{1,0 S_{\phi}}<\frac{\varepsilon_{\phi}}{6} .
$$

2. Now let $S \in\left(0, S_{\phi}\right]$ be given. For every $\chi \in W_{\phi}$ and $t \in[0, S]$,

$$
\begin{aligned}
A_{S}(\chi, 0)(t) & =\int_{0}^{t}\left(f\left(\left(P_{S} \chi\right)_{w}\right)-f(\chi)\right) d w \\
& =\int_{0}^{t}\left(f\left(\left(P_{S_{\phi}} \chi\right)_{w}\right)-f(\chi)\right) d w=A_{S_{\phi}}(\chi, 0)(t) .
\end{aligned}
$$

Using this (for $\chi$ and $\phi$ ) and the definition of the norms $|\cdot|_{1,0 S},|\cdot|_{1,0 S_{\phi}}$, one gets

$$
\left|A_{S}(\chi, 0)-A_{S}(\phi, 0)\right|_{1,0 S} \leq\left|A_{S_{\phi}}(\chi, 0)-A_{S_{\phi}}(\phi, 0)\right|_{1,0 S_{\phi}}<\frac{\varepsilon_{\phi}}{6}
$$

for every $\chi \in W_{\phi}$.

3. Let $\chi \in W_{\phi}, \eta \in C_{0 S, 0}^{1}, \widetilde{\eta} \in C_{0 S, 0}^{1}$ be given, with $|\eta|_{1,0 S} \leq \varepsilon_{\phi} / 2$ and $|\widetilde{\eta}|_{1,0 S} \leq \varepsilon_{\phi} / 2$. Proposition 4.1 yields

$$
\begin{aligned}
\left|A_{S}(\chi, \widetilde{\eta})-A_{S}(\chi, \eta)\right|_{1,0 S} & \leq 2 j S(1+S)|\widetilde{\eta}-\eta|_{1,0 S} \\
& \leq 2 j S_{\phi}\left(1+S_{\phi}\right)|\widetilde{\eta}-\eta|_{1,0 S} \leq \min \left\{\frac{1}{2}, \frac{\varepsilon_{\phi}}{3}\right\}|\widetilde{\eta}-\eta|_{1,0 S} .
\end{aligned}
$$

Furthermore,

$$
\begin{aligned}
\left|A_{S}(\chi, \eta)\right|_{1,0 S} & \\
& \leq\left|A_{S}(\chi, \eta)-A_{S}(\chi, 0)\right|_{1,0 S}+\left|A_{S}(\chi, 0)-A_{S}(\phi, 0)\right|_{1,0 S}+\left|A_{S}(\phi, 0)\right|_{1,0 S} \\
& <\frac{\varepsilon_{\phi}}{3}|\eta|_{1,0 S}+\frac{\varepsilon_{\phi}}{6}+\frac{\varepsilon_{\phi}}{6} \leq \frac{\varepsilon_{\phi}}{3} \frac{\varepsilon_{\phi}}{2}+\frac{2 \varepsilon_{\phi}}{6}=\frac{\varepsilon_{\phi}}{2} .
\end{aligned}
$$

For each $S \in\left(0, S_{\phi}\right]$ now Theorem 7.2 applies to the map

$$
W_{\phi} \times\left\{\eta \in C_{0 S, 0}^{1}:|\eta|_{1,0 S}<\varepsilon_{\phi}\right\} \ni(\chi, \eta) \mapsto A_{S}(\chi, \eta) \in C_{0 S, 0}^{1},
$$

with $M=M_{\phi}=\left\{\eta \in C_{0 S, 0}^{1}:|\eta|_{1,0 S} \leq \varepsilon_{\phi} / 2\right\}$, and yields a continuously differentiable map

$$
W_{\phi} \ni \chi \mapsto \eta_{\chi} \in C_{0 S, 0}^{1}
$$

given by $\eta_{\chi} \in M_{\phi}$ and $A_{S}\left(\chi, \eta_{\chi}\right)=\eta_{\chi}$. As the maps $P_{S}$ and $Z_{S}$ are linear and continuous, it follows that the map

$$
\Sigma_{\phi}: W_{\phi} \ni \chi \mapsto P_{S} \chi+Z_{S} \eta_{\chi} \in C_{S}^{1}
$$

is continuously differentiable. 
Using this and the continuous linear maps $E_{S}^{1}(\cdot, t): C_{S}^{1} \rightarrow C^{1}, 0 \leq t \leq S$, one gets that each map

$$
W_{\phi} \ni \chi \mapsto E_{S}^{1}\left(\Sigma_{\phi}(\chi), t\right) \in C^{1}, \quad 0 \leq t \leq S,
$$

is continuously differentiable. The map

$$
[0, S] \times W_{\phi} \ni(t, \chi) \mapsto E_{S}^{1}\left(\Sigma_{\phi}(\chi), t\right) \in C^{1}
$$

is continuous.

Proposition 4.4. For every $S \in\left(0, S_{\phi}\right]$ and for every $\chi \in W_{\phi} \cap X_{f}$ the function $x=x^{(\chi)}=\Sigma_{\phi}(\chi)$ is a solution of (1.1) on $[0, S]$, with $x_{0}=\chi$ and $x_{t} \in X_{f}$ for $0 \leq t \leq S$.

Proof. The function $x$ is continuously differentiable with $x_{0}=\left(P_{S} \chi\right)_{0}+$ $\left(Z_{S} \eta_{\chi}\right)_{0}=\left(P_{S} \chi\right)_{0}=\chi$, and for $0 \leq t \leq S$,

$$
\begin{aligned}
x(t) & =P_{S} \chi(t)+Z_{S} \eta_{\chi}(t)=\chi(0)+t \chi^{\prime}(0)+\eta_{\chi}(t) \\
& =x(0)+t f(\chi)+A_{S}\left(\chi, \eta_{\chi}\right)(t)
\end{aligned}
$$

(with $\chi \in X_{f}$ and the fixed point equation)

$$
\begin{aligned}
& =x(0)+t f(\chi)+\int_{0}^{t}\left(f\left(\left(P_{S} \chi\right)_{w}+\left(Z_{S} \eta_{\chi}\right)_{w}\right)-f(\chi)\right) d w \\
& =x(0)+\int_{0}^{t} f\left(x_{w}\right) d w .
\end{aligned}
$$

Observe that the restrictions of the maps $E_{S}^{1}\left(\Sigma_{\phi}(\cdot), t\right), 0 \leq t \leq S$, to the open subset $W_{\phi} \cap X_{f}$ of the manifold $X_{f}$ are continuously differentiable, and the restriction of the map $E_{S}^{1}\left(\Sigma_{\phi}(\cdot), \cdot\right)$ to $[0, S] \times\left(W_{\phi} \cap X_{f}\right)$ is continuous. In other words, the map

$$
[0, S] \times\left(W_{\phi} \cap X_{f}\right) \ni(t, \chi) \mapsto x_{t}^{(\chi)} \in X_{f}
$$

is continuous and each map

$$
W_{\phi} \cap X_{f} \ni \chi \mapsto x_{t}^{(\chi)} \in X_{f}, \quad 0 \leq t \leq S,
$$

is continuously differentiable.

Proposition 4.5 (Local uniqueness). Suppose $x$ and $\widetilde{x}$ are solutions of (1.1) on an interval $I$ of positive length, with $\min I=0$ and $x_{0}=\widetilde{x}_{0} \in X_{f}$. Then there exists $\tau>0$ with $x(t)=\widetilde{x}(t)$ for all $t \leq \tau$.

Proof. Let $\phi=x_{0}$ and consider $T_{\phi}, \varepsilon_{\phi}, S_{\phi}$ as in Proposition 4.3. By continuity, there exists $\tau=S \in\left(0, S_{\phi}\right] \cap I$ such that for $0 \leq t \leq S$,

$$
\begin{array}{lll}
\left|x(t)-\phi(0)-t \phi^{\prime}(0)\right|<\frac{\varepsilon_{\phi}}{4}, & & \left|x^{\prime}(t)-\phi^{\prime}(0)\right|<\frac{\varepsilon_{\phi}}{4}, \\
\left|\widetilde{x}(t)-\phi(0)-t \phi^{\prime}(0)\right|<\frac{\varepsilon_{\phi}}{4}, & \left|\widetilde{x}^{\prime}(t)-\phi^{\prime}(0)\right|<\frac{\varepsilon_{\phi}}{4} .
\end{array}
$$


Define

$$
\begin{array}{ll}
y=\left.x\right|_{(-\infty, S]}-P_{S} \phi, & \eta=\left.y\right|_{[0, S]} \in C_{0 S, 0}^{1}, \\
\widetilde{y}=\left.\widetilde{x}\right|_{(-\infty, S]}-P_{S} \phi, & \widetilde{\eta}=\left.\widetilde{y}\right|_{[0, S]} \in C_{0 S, 0}^{1} .
\end{array}
$$

Then $|\eta|_{1,0 S}<\varepsilon_{\phi} / 2$ and $|\widetilde{\eta}|_{1,0 S}<\varepsilon_{\phi} / 2$.

By Proposition $4.3,(\phi, \eta) \in D_{S}$ and $(\phi, \widetilde{\eta}) \in D_{S}$. Also, for $0 \leq t \leq S$,

$$
\begin{aligned}
A_{S}(\phi, \eta)(t) & =\int_{0}^{t}\left(f\left(\left(P_{S} \phi\right)_{w}+\left(Z_{S} \eta\right)_{w}\right)-f(\phi)\right) d w \\
& =\int_{0}^{t} f\left(x_{w}\right) d w-t f(\phi)=x(0)+\int_{0}^{t} f\left(x_{w}\right) d w-\left(\phi(0)+t \phi^{\prime}(0)\right) \\
& =x(t)-\left(P_{S} \phi\right)(t)=\eta(t)
\end{aligned}
$$

(recall that Proposition 3.1 yields the continuity of $(-\infty, S] \ni w \mapsto x_{w}=$ $\left.E_{S}^{1}\left(\left.x\right|_{(-\infty, S]}, w\right) \in C^{1}\right)$. Hence $A_{S}(\phi, \eta)=\eta$. Analogously, $A_{S}(\phi, \widetilde{\eta})=\widetilde{\eta}$. Proposition 4.3 yields

$$
|\eta-\widetilde{\eta}|_{1,0 S}=\left|A_{S}(\phi, \eta)-A_{S}(\phi, \widetilde{\eta})\right|_{1,0 S} \leq \frac{1}{2}|\eta-\widetilde{\eta}|_{1,0 S},
$$

which gives $\eta=\widetilde{\eta}$ and thereby $x(t)=\widetilde{x}(t)$ on $[0, S]=[0, \tau]$.

\section{The semiflow on the solution manifold}

Proposition 5.1. Suppose $x$ and $\widetilde{x}$ are solutions of (1.1) on intervals $I$ and $\widetilde{I}$ of positive length, and $0=\min I=\min \widetilde{I}, x_{0}=\widetilde{x}_{0} \in X_{f}$. Then $x(t)=\widetilde{x}(t)$ on $I \cap \widetilde{I}$.

Proof. The interval $J=I \cap \widetilde{I}$ has positive length and $\min J=0$. Assume $x(t) \neq \widetilde{x}(t)$ for some $t \in J$. Set $t_{J}=\inf \{t \in J: x(t) \neq \widetilde{x}(t)\}$ and assume in addition that $t_{J}<\sup J$. The continuously differentiable function $y:\left(-\infty, \sup J-t_{J}\right) \rightarrow \mathbb{R}^{n}$ given by $y(t)=x\left(t+t_{J}\right)$ satisfies

$$
y^{\prime}(t)=x^{\prime}\left(t+t_{J}\right)=f\left(x_{t+t_{J}}\right)=f\left(y_{t}\right)
$$

for $0 \leq t<\sup J-t_{J}$, in particular, $y_{0} \in X_{f}$. Analogously, the function $\widetilde{y}:\left(-\infty, \sup J-t_{J}\right) \rightarrow \mathbb{R}^{n}$ given by $y(t)=\widetilde{x}\left(t+t_{J}\right)$ is a solution of equation (1.1) on $\left[0, \sup J-t_{J}\right)$, and $y_{0}=\widetilde{y}_{0}$. Proposition 4.5 yields $y(t)=\widetilde{y}(t)$ on $[0, \tau]$ for some $\tau>0$. This implies $x(t)=\widetilde{x}(t)$ on $\left[t_{J}, t_{J}+\tau\right]$, contradicting the definition of $t_{J}$. It follows that $t_{J}=\sup J$, hence $x(t)=\widetilde{x}(t)$ on $[0, \sup J)$. In case $\sup J=\max J$ continuity yields $x(t)=\widetilde{x}(t)$ on $J$. In both cases, one arrives at a contradiction to the first assumption.

Now maximal solutions are defined as follows. Let $\phi \in X_{f}$. Set

$t_{\phi}=\sup \left\{t>0:\right.$ there is a solution of (1.1) on $[0, t]$ with $\left.x_{0}=\phi\right\} \leq \infty$. 
By Proposition 4.4, $0<t_{\phi}$. Using Proposition 5.1, one obtains a solution $x^{\phi}$ of equation (1.1) on $\left[0, t_{\phi}\right)$, with $x_{0}^{\phi}=\phi$, by $x^{\phi}(t)=x(t)$ for $0<t<t_{\phi}$, where $x$ is any solution of equation (1.1) on $\left[0, t^{\prime}\right]$ with $t<t^{\prime}<t_{\phi}$ and $x_{0}=\phi$. Equation (1.1) yields $x_{t}^{\phi} \in X_{f}$ for $0 \leq t<t_{\phi}$.

It is easy to show that any solution of (1.1) on some interval $I$ of positive length with $\min I=0$ and $x_{0}=\phi$ is a restriction of $x^{\phi}$.

Set $\Omega_{f}=\left\{(t, \phi) \in[0, \infty) \times X_{f}: t<t_{\phi}\right\}$ and define $\Sigma_{f}: \Omega_{f} \rightarrow X_{f}$ by $\Sigma_{f}(t, \phi)=x_{t}^{\phi}$.

Proposition 5.2. The map $\Sigma_{f}$ is a semiflow.

Proof. For every $\phi \in X_{f}, 0<t_{\phi}$, hence $(0, \phi) \in \Omega_{f}$ and $\Sigma_{f}(0, \phi)=x_{0}^{\phi}=\phi$. Let $(t, \phi) \in \Omega_{f}$ and $(s, \Sigma(t, \phi)) \in \Omega_{f}$. It remains to show that $(t+s, \phi) \in \Omega_{f}$ and $\Sigma_{f}(s+t, \phi)=\Sigma_{f}\left(s, \Sigma_{f}(t, \phi)\right)$. In order to prove this let $x=x^{\phi}, \psi=x_{t}, y=x^{\psi}$. Define $\xi:(-\infty, s+t] \rightarrow \mathbb{R}^{n}$ by $\xi(u)=y(u-t)$. The map $\xi$ is continuously differentiable, and for $u \leq t$,

$$
\xi(u)=y(u-t)=\psi(u-t)=x_{t}(u-t)=x(u) .
$$

In particular, $\xi_{0}=\phi$ and $\xi^{\prime}(u)=f\left(\xi_{u}\right)$ for $0 \leq u \leq t$. For $t<u \leq t+s$,

$$
\xi^{\prime}(u)=y^{\prime}(u-t)=f\left(y_{u-t}\right)=f\left(\xi_{u}\right) .
$$

It follows that $\xi$ is a restriction of $x^{\phi}$. Hence $s+t<t_{\phi}$, or, $(s+t, \phi) \in \Omega_{f}$, and

$$
\Sigma_{f}(s+t, \phi)=\xi_{s+t}=y_{s}=\Sigma_{f}(s, \psi)=\Sigma_{f}\left(s, \Sigma_{f}(t, \phi)\right) .
$$

For $t \geq 0$ with $\Omega_{f, t}=\left\{\phi \in X_{f}:(t, \phi) \in \Omega_{f}\right\} \neq \emptyset$ consider the solution operator $\Sigma_{f, t}: \Omega_{f, t} \rightarrow X_{f}$ given by $\Sigma_{f, t}(\phi)=\Sigma_{f}(t, \phi)$.

Proposition 5.3. For every $(t, \phi) \in \Omega_{f}$ there exist an open neighbourhood $N$ of $\phi$ in $X_{f}$ and $\varepsilon>0$ with $[0, t+\varepsilon) \times N \subset \Omega_{f},\left.\Sigma_{f}\right|_{[0, t+\varepsilon) \times N}$ continuous, and $\left.\Sigma_{f, t}\right|_{N}$ continuously differentiable.

Proof. 1. Let $(t, \phi) \in \Omega_{f}$ be given. The remarks following Proposition 4.4 show that $t=0$ is contained in the set

$A=\left\{s \in\left[0, t_{\phi}\right):\right.$ there exist an open neighbourhood $V_{s}$ of $\phi$ in $X_{f}$ and $\varepsilon_{s}>0$ with $\left[0, s+\varepsilon_{s}\right) \times V_{s} \subset \Omega_{f},\left.\Sigma_{f}\right|_{\left[0, s+\varepsilon_{s}\right) \times V_{s}}$ continuous, and $\left.\Sigma_{f, s}\right|_{V_{s}}$ continuously differentiable\}.

Let $t_{A}=\sup A \leq t_{\phi}$. It remains to prove that $t_{A}=t_{\phi}$.

2. Suppose $t_{A}<t_{\phi}$. Set $\psi=\Sigma_{f}\left(t_{A}, \phi\right)$. Again by the remarks following Proposition 4.4, there exist an open neighbourhood $W$ of $\psi$ in $X_{f}$ and $\tau>0$ with $[0, \tau] \times W \subset \Omega_{f}$ so that $\left.\Sigma_{f}\right|_{[0, \tau] \times W}$ is continuous and all $\left.\Sigma_{f, u}\right|_{W}, 0 \leq u \leq \tau$, are continuously differentiable. Proposition 3.1 (a) yields that the flowline $\left[0, t_{\phi}\right) \ni$ 
$s \mapsto x_{s}^{\phi} \in X_{f}$ is continuous (observe $x_{s}^{\phi}=E_{u}^{1}\left(\left.x^{\phi}\right|_{(-\infty, u]}, s\right)$ for $\left.0 \leq s<u<t_{\phi}\right)$. It follows that there exists

$$
t_{0} \in A \cap\left(t_{A}-\frac{\tau}{2}, t_{A}\right) \quad \text { with } x_{t_{0}}^{\phi} \in W .
$$

From $t_{0} \in A$ one obtains an open neighbourhood $N_{0}$ of $\phi$ in $X_{f}$ and $\varepsilon_{0}>0$ so that $\left[0, t_{0}+\varepsilon_{0}\right) \times N_{0} \subset \Omega_{f}$, and $\left.\Sigma_{f}\right|_{\left[0, t_{0}+\varepsilon_{0}\right) \times N_{0}}$ is continuous, and $\left.\Sigma_{f, t_{0}}\right|_{N_{0}}$ is continuously differentiable. Because of continuity and $x_{t_{0}}^{\phi} \in W$ one may assume $\Sigma_{f, t_{0}}\left(N_{0}\right) \subset W$. For $t_{0}<u<t_{A}+\tau / 2$ and $\chi \in N_{0}$,

$$
0<u-t_{0}<\tau \text { and } \Sigma_{f, t_{0}}(\chi) \in W,
$$

which gives $(u, \chi)=\left(\left(u-t_{0}\right)+t_{0}, \chi\right) \in \Omega_{f}$ and

$$
\Sigma_{f}(u, \chi)=\Sigma_{f}\left(u-t_{0}, \Sigma_{f}\left(t_{0}, \chi\right)\right) .
$$

It follows that $\left.\Sigma_{f}\right|_{\left(t_{0}, t_{A}+\tau / 2\right) \times N_{0}}$ is continuous, which in combination with the continuity of the restriction $\left.\Sigma_{f}\right|_{\left[0, t_{0}+\varepsilon_{0}\right) \times N_{0}}$ yields that the restriction of $\Sigma_{f}$ to $\left[0, t_{A}+\tau / 2\right) \times N_{0}$ is continuous.

3. For $u=t_{A}+\tau / 4$ and $\chi \in N_{0}$,

$$
\Sigma_{f}(u, \chi)=\Sigma_{f}\left(u-t_{0}, \Sigma_{f}\left(t_{0}, \chi\right)\right)=\Sigma_{f, u-t_{0}} \circ \Sigma_{f, t_{0}}(\chi)
$$

with $0<u-t_{0}<\tau$. Recall that $\Sigma_{f, t_{0}}\left(N_{0}\right) \subset W$. Now it follows that $\left.\Sigma_{f, u}\right|_{N_{0}}$ is continuously differentiable. Combining this with the result of part 2 of the proof, one concludes that $u>t_{A}$ belongs to $A$, contradicting $t_{A}=\sup A$.

Corollary 5.4. The semiflow $\Sigma_{f}$ is continuous, each set $\Omega_{f, t}, t \geq 0$, is open in $X_{f}$, and each solution operator $\Sigma_{f, t}, t \geq 0$, and $\Omega_{f, t} \neq \emptyset$, is continuously differentiable.

Proof. Let $t \geq 0$ and $\phi \in \Omega_{f, t}$ be given. Then $(t, \phi) \in \Omega_{f}$, and for $N$ chosen according to Proposition 5.3 we get $N \subset \Omega_{f, t}$. This shows that $\Omega_{f, t}$ is an open subset of $X_{f}$. The remaining assertions are obvious from Proposition 5.4.

The next result on the derivatives $D \Sigma_{f, t}(\phi), \phi \in \Omega_{f, t}$, will be used in Section 6 .

Proposition 5.5. Let $\phi \in X_{f}, 0 \leq t<t_{\phi}, \widehat{\phi} \in T_{\phi} X_{f}$, and $s \leq 0$. Then

$$
\begin{array}{ll}
\left(D \Sigma_{f, t}(\phi) \widehat{\phi}\right)(s)=\widehat{\phi}(t+s) & \text { in case } t+s \leq 0, \\
\left(D \Sigma_{f, t}(\phi) \widehat{\phi}\right)(s)=\left(D \Sigma_{f, t+s}(\phi) \widehat{\phi}\right)(0) & \text { in case } 0 \leq t+s .
\end{array}
$$

Proof. Each linear map ev ${ }_{1, s}: C^{1} \ni \psi \mapsto \psi(s) \in \mathbb{R}^{n}, s \leq 0$, is continuous (compare Proposition 2.1). Let $\phi \in X_{f}, 0 \leq t<t_{\phi}, \widehat{\phi} \in T_{\phi} X_{f}, s \leq 0$. Then

$$
\begin{aligned}
\left(D \Sigma_{f, t}(\phi) \widehat{\phi}\right)(s) & =\operatorname{ev}_{1, s}\left(D \Sigma_{f, t}(\phi) \widehat{\phi}\right)=D\left(\mathrm{ev}_{1, s} \circ \Sigma_{f, t}\right)(\phi) \widehat{\phi} \\
& =D\left\{\Omega_{f, t} \ni \widetilde{\phi} \mapsto x_{t}^{\widetilde{\phi}}(s) \in \mathbb{R}^{n}\right\}(\phi) \widehat{\phi} \\
& =D\left\{\Omega_{f, t} \ni \widetilde{\phi} \mapsto x^{\widetilde{\phi}}(t+s) \in \mathbb{R}^{n}\right\}(\phi) \widehat{\phi} .
\end{aligned}
$$


In case $0 \leq t+s$ the set $\Omega_{f, t} \subset \Omega_{f, t+s}$ is an open neighbourhood of $\phi$ in $X_{f}$, and

$$
\begin{aligned}
D\left\{\Omega_{f, t} \ni \widetilde{\phi} \mapsto x^{\widetilde{\phi}}(t+s) \in \mathbb{R}^{n}\right\}(\phi) \widehat{\phi} & =D\left\{\Omega_{f, t} \ni \widetilde{\phi} \mapsto x_{t+s}^{\widetilde{\phi}}(0) \in \mathbb{R}^{n}\right\}(\phi) \widehat{\phi} \\
& =D\left(\operatorname{ev}_{1,0} \circ \Sigma_{f, t+s}\right)(\phi) \widehat{\phi} \\
& =\operatorname{ev}_{1,0}\left(D \Sigma_{f, t+s}(\phi) \widehat{\phi}\right)=\left(D \Sigma_{f, t+s}(\phi) \widehat{\phi}\right)(0)
\end{aligned}
$$

while in case $t+s \leq 0$

$$
\begin{aligned}
D\left\{\Omega_{f, t} \ni \widetilde{\phi} \mapsto x^{\widetilde{\phi}}(t+s) \in \mathbb{R}^{n}\right\}(\phi) \widehat{\phi} & =D\left\{\Omega_{f, t} \ni \widetilde{\phi} \mapsto \widetilde{\phi}(t+s) \in \mathbb{R}^{n}\right\}(\phi) \widehat{\phi} \\
& =D \operatorname{ev}_{1, t+s}(\phi) \widehat{\phi}=\operatorname{ev}_{1, t+s}(\widehat{\phi})=\widehat{\phi}(t+s) .
\end{aligned}
$$

\section{The variational equation}

For $\phi \in X_{f}$ the derivatives $D \Sigma_{f, t}(\phi): T_{\phi} X_{f} \rightarrow T_{\Sigma_{f, t}(\phi)} X_{f}, 0 \leq t<t_{\phi}$, are given by a variational equation. In order to prove this let $\widehat{\phi} \in T_{\phi} X_{f}$ and define the map $v^{\phi, \widehat{\phi}}:\left(-\infty, t_{\phi}\right) \rightarrow \mathbb{R}^{n}$ by

$$
\begin{aligned}
& v^{\phi, \widehat{\phi}}(t)=\left(D \Sigma_{f, t}(\phi) \widehat{\phi}\right)(0) \quad \text { for } 0 \leq t<t_{\phi}, \\
& v^{\phi, \widehat{\phi}}(t)=\widehat{\phi}(t) \quad \text { for } t<0 .
\end{aligned}
$$

Proposition 6.1. Let $\phi \in X_{f}$ and $\widehat{\phi} \in T_{\phi} X_{f}$ be given and consider the map $v=v^{\phi, \widehat{\phi}}$.

(a) $v_{t}=D \Sigma_{f, t}(\phi) \widehat{\phi}$ for every $t \in\left[0, t_{\phi}\right)$.

In particular, $v_{0}=\widehat{\phi}$. The map $v:\left(-\infty, t_{\phi}\right) \rightarrow \mathbb{R}^{n}$ is continuously differentiable and the curve $\left[0, t_{\phi}\right) \ni t \mapsto v_{t} \in C^{1}$ is continuous, and

(b) $v^{\prime}(t)=D f\left(x_{t}^{\phi}\right) v_{t}$ for every $t \in\left[0, t_{\phi}\right)$.

Proof. 1. (a) Let $\phi \in X_{f}, \widehat{\phi} \in T_{\phi} X_{f}, 0 \leq t<t_{\phi}$. For $s \leq 0$ with $0 \leq t+s$ Proposition 5.5 yields

$$
v_{t}(s)=v(t+s)=\left(D \Sigma_{f, t+s}(\phi) \widehat{\phi}\right)(0)=\left(D \Sigma_{f, t}(\phi) \widehat{\phi}\right)(s),
$$

and, for $s \leq 0$ with $t+s<0$,

$$
v_{t}(s)=v(t+s)=\widehat{\phi}(t+s)=\left(D \Sigma_{f, t}(\phi) \widehat{\phi}\right)(s) .
$$

Together, $v_{t}=D \Sigma_{f, t}(\phi) \widehat{\phi}$. Notice that $D \Sigma_{f, 0}(\phi) \widehat{\phi}=\widehat{\phi}$. The fact that each $v_{t}=D \Sigma_{f, t}(\phi) \widehat{\phi}, 0 \leq t<t_{\phi}$, belongs to $T_{\Sigma_{f, t}(\phi)} X_{f} \subset C^{1}$ implies that $v$ is continuously differentiable. Using Proposition 3.1 (a), one obtains that the curve $\left[0, t_{\phi}\right) \ni t \mapsto v_{t} \in C^{1}$ is continuous.

2. Let $t>0$ with $\Omega_{f, t} \neq \emptyset$ be given. For $\phi \in \Omega_{f, t}$ consider the map

$$
\eta^{\phi}:[0, t] \ni s \mapsto x^{\phi}(s)-\phi(0)-s \phi^{\prime}(0) \in \mathbb{R}^{n} .
$$

Observe that $\eta^{\phi} \in C_{0 t, 0}^{1}$ and $P_{t} \phi+Z_{t} \eta^{\phi}=\left.x^{\phi}\right|_{(-\infty, t]}$, which yields

$$
\left(P_{t} \phi+Z_{t} \eta^{\phi}\right)_{s}=x_{s}^{\phi} \in X_{f} \subset U \text { for } 0 \leq s \leq t .
$$


It follows that $P_{t} \phi+Z_{t} \eta^{\phi} \in \operatorname{dom}_{t}$. Then $\left(\phi, \eta^{\phi}\right)$ belongs to the domain $D_{t}$ of the map $G_{t}$. The map $Y_{t}: \Omega_{f, t} \ni \phi \mapsto \eta^{\phi} \in C_{0 t, 0}^{1}$ satisfies

$$
\begin{aligned}
Y_{t}(\phi)(s) & =\eta^{\phi}(s)=x^{\phi}(s)-\phi(0)-s \phi^{\prime}(0) \\
& =\int_{0}^{s} f\left(x_{u}^{\phi}\right) d u-s f(\phi)=\int_{0}^{s}\left(f\left(\left(P_{t} \phi+Z_{t} \eta^{\phi}\right)_{u}\right)-f(\phi)\right) d u \\
& =\int_{0}^{s}\left(f\left(E_{t}^{1}\left(P_{t} \phi+Z_{t} Y_{t}(\phi), u\right)\right)-f(\phi)\right) d u=I_{t}\left(G_{t}\left(\phi, Y_{t}(\phi)\right)\right)(s)
\end{aligned}
$$

for all $\phi \in \Omega_{f, t}$ and $s \in[0, t]$, hence

$$
Y_{t}(\phi)=I_{t}\left(G_{t}\left(\phi, Y_{t}(\phi)\right)\right) \quad\left(=A_{t}\left(\phi, Y_{t}(\phi)\right)\right) \quad \text { for all } \quad \phi \in \Omega_{f, t} .
$$

3. Proof that the map $Y$ is continuously differentiable with

$$
v^{\phi, \widehat{\phi}}(s)=D Y_{t}(\phi) \widehat{\phi}(s)+\left(P_{t} \widehat{\phi}\right)(s) \quad \text { for all } s \in[0, t], \phi \in \Omega_{f, t}, \widehat{\phi} \in T_{\phi} X_{f} \text {. }
$$

By part $2,\left(\phi, Y_{t}(\phi)\right) \in D_{t}$ for all $\phi \in \Omega_{f, t}$. With the shift map $\Delta_{t}: C^{1} \rightarrow C_{t}^{1}$, $\Delta_{t} \phi(s)=\phi(s-t)$ and the restriction map $R_{t}: C_{t}^{1} \rightarrow C_{0 t}^{1}, R_{t} \chi=\left.\chi\right|_{[0, t]}$, which are both linear and continuous,

$$
Y_{t}(\phi)=R_{t}\left(\Delta_{t} \circ \Sigma_{f, t}(\phi)-P_{t} \phi\right) \quad \text { for all } \phi \in \Omega_{f, t} .
$$

This shows that the map $Y_{t}$ is continuously differentiable, and for all $\phi \in \Omega_{f, t}$, $\widehat{\phi} \in T_{\phi} X_{f}, s \in[0, t]$,

$$
\begin{aligned}
\left(D Y_{t}(\phi) \widehat{\phi}\right)(s) & =\left(R_{t} \Delta_{t} D \Sigma_{f, t}(\phi) \widehat{\phi}\right)(s)-\left(R_{t} P_{t} \widehat{\phi}\right)(s) \\
& =\left(D \Sigma_{f, t}(\phi) \widehat{\phi}\right)(s-t)-\widehat{\phi}(0)-s \widehat{\phi}^{\prime}(0) \\
& =\left(D \Sigma_{f, s}(\phi) \widehat{\phi}\right)(0)-\widehat{\phi}(0)-s \widehat{\phi}^{\prime}(0) \quad(\text { see Proposition 5.5) } \\
& =v^{\phi, \widehat{\phi}}(s)-\widehat{\phi}(0)-s \widehat{\phi}^{\prime}(0)=v^{\phi, \widehat{\phi}}(s)-P_{t} \widehat{\phi}(s) .
\end{aligned}
$$

4. Differentiation of equation (6.1) yields

(6.2) $D Y_{t}(\phi) \widehat{\phi}=I_{t} D G_{t}\left(\phi, Y_{t}(\phi)\right)\left(\widehat{\phi}, D Y_{t}(\phi) \widehat{\phi}\right) \quad$ for all $\phi \in \Omega_{f, t}, \widehat{\phi} \in T_{\phi} X_{f}$.

For such $\phi$ and $\widehat{\phi}$ and for each $s \in[0, t]$,

$$
\begin{aligned}
v^{\phi, \widehat{\phi}}(s)= & \left(D Y_{t}(\phi) \widehat{\phi}\right)(s)+\widehat{\phi}(0)+s \widehat{\phi}^{\prime}(0) \quad(\text { see part 3) } \\
= & \int_{0}^{s}\left\{D_{\mathrm{e}} f\left(\left(P_{t} \phi\right)_{u}+\left(Z_{t} Y_{t}(\phi)\right)_{u}\right)\left(\left(P_{t} \widehat{\phi}\right)_{u}+\left(Z_{t} D Y_{t}(\phi) \widehat{\phi}\right)_{u}\right)-D f(\phi) \widehat{\phi}\right\} d u \\
& +\widehat{\phi}(0)+s \widehat{\phi}^{\prime}(0) \quad(\text { with }(6.2) \text { and Corollary 3.3) } \\
= & \left.\int_{0}^{s}\left\{D_{\mathrm{e}} f\left(x_{u}^{\phi}\right)\left(v_{u}^{\phi, \widehat{\phi}}\right)-D f(\phi) \widehat{\phi}\right\} d u+\widehat{\phi}(0)+s D f(\phi) \widehat{\phi} \quad \text { as } \widehat{\phi} \in T_{\phi} X_{f}\right) \\
= & \int_{0}^{s} D f\left(x_{u}^{\phi}\right) v_{u}^{\phi, \widehat{\phi}} d u+\widehat{\phi}(0) .
\end{aligned}
$$

In case $0<t$ differentiation yields $\left(v^{\phi, \widehat{\phi}}\right)^{\prime}(t)=D f\left(x_{t}^{\phi}\right) v_{t}^{\phi, \widehat{\phi}}$. By continuity, this holds for $t=0$ as well. 


\section{Appendix on parametrized contractions and implicit functions}

Proposition 7.1. Let a Hausdorff space $T$, a complete metric space $M$, and a map $f: T \times M \rightarrow M$ be given. Assume that $f$ is a uniform contraction in the sense that there exists $k \in[0,1)$ so that

$$
d(f(t, x), f(t, y)) \leq k d(x, y) \quad \text { for all } t \in T, x \in M, y \in M,
$$

and $f(\cdot, x): T \rightarrow M$ is continuous for each $x \in M$. Then the map $g: T \rightarrow M$ given by $g(t)=f(t, g(t))$ is continuous.

TheOREM 7.2. Let a Fréchet space $T$, a Banach space $B$, open sets $V \subset T$ and $O_{B} \subset B$, and a continuously differentiable map $A: V \times O_{B} \rightarrow B$ be given. Assume that for a closed set $M \subset O_{B}$ we have $A(V \times M) \subset M$, and $A$ is a uniform contraction in the sense that there exists $k \in[0,1)$ so that

$$
|A(t, x)-A(t, y)| \leq k|x-y| \quad \text { for all } t \in V, x \in O_{B}, y \in O_{B} .
$$

Then the map $g: V \rightarrow B$ given by $g(t)=A(t, g(t)) \in M$ is continuously differentiable.

Proof. 1. Continuous differentiability (in the Michal-Bastiani sense) implies continuity. So Proposition 7.1 applies to the restriction of $A$ to $V \times M$ and yields a continuous map $g: V \rightarrow B$ with $g(t)=A(t, g(t)) \in M$ for all $t \in V$. Choose $\kappa \in(k, 1)$. Each linear map $D_{2} A(t, x): B \rightarrow B,(t, x) \in V \times O_{B}$, is continuous. The contraction property yields

$$
\left|D_{2} A(t, x)\right| \leq \kappa \quad \text { for all }(t, x) \in V \times O_{B}
$$

since given $\varepsilon=\kappa-k$ and $t \in V, x \in O_{B}$, and $\widehat{x} \in B$ with $|\widehat{x}| \leq 1$ there exists $\delta>0$ such that, for $h=\delta / 2, x+h \widehat{x} \in O_{B}$ and

$$
\begin{aligned}
\left|h^{-1}(A(t, x)-A(t, x+h \widehat{x}))-D_{2} A(t, x) \widehat{x}\right| \\
\quad=\left|h^{-1}(A(t, x)-A(t, x+h \widehat{x}))-D A(t, x)(0, \widehat{x})\right| \leq \varepsilon,
\end{aligned}
$$

hence

$$
\begin{aligned}
|h|\left|D_{2} A(t, x) \widehat{x}\right| & \leq \varepsilon|h|+|A(t, x+h \widehat{x})-A(t, x)| \\
& \leq \varepsilon|h|+k|h \widehat{x}| \leq(\varepsilon+k)|h|=\kappa|h| .
\end{aligned}
$$

It follows that $\left|D_{2} A(t, x)\right|=\sup _{|\widehat{x}| \leq 1}\left|D_{2} A(t, x) \widehat{x}\right| \leq \kappa$, for every $(t, x) \in V \times O_{B}$.

2. The map $\alpha: V \times O_{B} \times T \times B \ni(t, x, \widehat{t}, \widehat{x}) \mapsto D A(t, x)(\widehat{t}, \widehat{x}) \in B$ is continuous, with

$$
\alpha(t, x, \widehat{t}, \widehat{x})=D_{1} A(t, x) \widehat{t}+D_{2} A(t, x) \widehat{x} .
$$

It follows that for all $(t, x, \widehat{t}) \in V \times O_{B} \times T$ and for all $\widehat{x}$ and $\widehat{y}$ in $B$,

$$
|\alpha(t, x, \widehat{t}, \widehat{x})-\alpha(t, x, \widehat{t}, \widehat{y})|=\left|D_{2} A(t, x)(\widehat{x}-\widehat{y})\right| \leq \kappa|\widehat{x}-\widehat{y}| .
$$


Therefore Proposition 7.1 yields a continuous map $\gamma: V \times O_{B} \times T \rightarrow B$ with

$$
\gamma(t, x, \widehat{t})=\alpha(t, x, \widehat{t}, \gamma(t, x, \widehat{t}))=D_{1} A(t, x) \widehat{t}+D_{2} A(t, x) \gamma(t, x, \widehat{t})
$$

for all $(t, x, \widehat{t}) \in V \times O_{B} \times T$. It follows that the map

$$
\xi: V \times T \ni(t, \widehat{t}) \mapsto \gamma(t, g(t), \widehat{t}) \in B
$$

is continuous.

3. It remains to show that for all $t \in V$ and all $\widehat{t} \in T$,

$$
\lim _{0 \neq h \rightarrow 0} h^{-1}(g(t+h \widehat{t})-g(t))=\xi(t, \widehat{t}),
$$

which in combination with the continuity of $\xi$ yields that $g$ is continuously differentiable, with $D g(t) \widehat{t}=\xi(t, \widehat{t})$. So let $t \in V$ and $\widehat{t} \in T$ be given. Choose a convex neighbourhood $N_{B} \subset O_{B}$ of $g(t)$. There exists $\delta>0$ such that, for $-\delta \leq h \leq \delta$,

$$
t+h \widehat{t} \in V \quad \text { and } \quad g(t+h \widehat{t}) \in N_{B} .
$$

Notice that, for all $h \in[-\delta, \delta]$ and all $\theta \in[0,1], g(t)+\theta(g(t+h \widehat{t})-g(t)) \in N_{B}$. With the abbreviation

$$
\begin{aligned}
\xi=\xi(t, \widehat{t}) & =\gamma(t, g(t), \widehat{t}) \\
& =D_{1} A(t, g(t)) \widehat{t}+D_{2} A(t, g(t)) \gamma(t, g(t), \widehat{t}) \\
& =D_{1} A(t, g(t)) \widehat{t}+D_{2} A(t, g(t)) \xi
\end{aligned}
$$

one finds that

$h^{-1}(g(t+h \widehat{t})-g(t))-\xi=h^{-1}(A(t+h \widehat{t}, g(t+h \widehat{t}))-A(t, g(t)))-\xi, \quad$ with $0<|h|<\delta$, equals

$$
\begin{aligned}
= & h^{-1}(A(t+h \widehat{t}, g(t+h \widehat{t}))-A(t+h \widehat{t}, g(t)))-D_{1} A(t, g(t)) \widehat{t}-D_{2} A(t, g(t)) \xi \\
& +h^{-1}(A(t+h \widehat{t}, g(t))-A(t, g(t))) \\
= & h^{-1}(A(t+h \widehat{t}, g(t))-A(t, g(t)))-D_{1} A(t, g(t)) \widehat{t} \\
& +h^{-1}(A(t+h \widehat{t}, g(t+h \widehat{t}))-A(t+h \widehat{t}, g(t))) \\
& -\int_{0}^{1} D_{2} A(t+h \widehat{t}, g(t)+\theta[g(t+h \widehat{t})-g(t)]) \xi d \theta \\
& +\int_{0}^{1}\left\{D_{2} A(t+h \widehat{t}, g(t)+\theta[g(t+h \widehat{t})-g(t)])-D_{2} A(t, g(t))\right\} \xi d \theta \\
= & h^{-1}(A(t+h \widehat{t}, g(t))-A(t, g(t)))-D_{1} A(t, g(t)) \widehat{t} \\
& +\int_{0}^{1} h^{-1} D_{2} A(t+h \widehat{t}, g(t)+\theta[g(t+h \widehat{t})-g(t)])[g(t+h \widehat{t})-g(t)] d \theta \\
& -\int_{0}^{1} D_{2} A(t+h \widehat{t}, g(t)+\theta[g(t+h \widehat{t})-g(t)]) \xi d \theta
\end{aligned}
$$




$$
\begin{aligned}
& +\int_{0}^{1}\left\{D_{2} A(t+h \widehat{t}, g(t)+\theta[g(t+h \widehat{t})-g(t)])-D_{2} A(t, g(t))\right\} \xi d \theta \\
= & h^{-1}(A(t+h \widehat{t}, g(t))-A(t, g(t)))-D_{1} A(t, g(t)) \widehat{t} \\
& +\int_{0}^{1} D_{2} A(t+h \widehat{t}, g(t)+\theta[g(t+h \widehat{t})-g(t)])\left[h^{-1}(g(t+h \widehat{t})-g(t))-\xi\right] d \theta \\
& +\int_{0}^{1}\left\{D_{2} A(t+h \widehat{t}, g(t)+\theta[g(t+h \widehat{t})-g(t)])-D_{2} A(t, g(t))\right\} \xi d \theta .
\end{aligned}
$$

Hence $\left|h^{-1}(g(t+h \widehat{t})-g(t))-\xi\right|$ is majorized by

$$
\begin{aligned}
\left|h^{-1}(A(t+h \widehat{t}, g(t))-A(t, g(t)))-D_{1} A(t, g(t)) \widehat{t}\right|+\kappa\left|h^{-1}(g(t+h \widehat{t})-g(t))-\xi\right| \\
+\left|\int_{0}^{1}\left\{D_{2} A(t+h \widehat{t}, g(t)+\theta[g(t+h \widehat{t})-g(t)])-D_{2} A(t, g(t))\right\} \xi d \theta\right|
\end{aligned}
$$

which yields

$$
\begin{aligned}
(1-\kappa) & \left|h^{-1}(g(t+h \widehat{t})-g(t))-\xi\right| \\
\leq & \left|h^{-1}(A(t+h \widehat{t}, g(t))-A(t, g(t)))-D_{1} A(t, g(t)) \widehat{t}\right| \\
& +\left|\int_{0}^{1}\left\{D_{2} A(t+h \widehat{t}, g(t)+\theta[g(t+h \widehat{t})-g(t)])-D_{2} A(t, g(t))\right\} \xi d \theta\right| .
\end{aligned}
$$

The first term in the last expression converges to 0 as $0 \neq h \rightarrow 0$. The map

$[-\delta, \delta] \times[0,1] \ni(h, \theta) \mapsto\left\{D_{2} A(t+h \widehat{t}, g(t)+\theta[g(t+h \widehat{t})-g(t)])-D_{2} A(t, g(t))\right\} \xi \in B$

is uniformly continuous with value 0 on $\{0\} \times[0,1]$. This implies that for $0 \neq$ $h \rightarrow 0$ the last integrand converges to 0 uniformly with respect to $\theta \in[0,1]$. Therefore the last integral tends to 0 as $0 \neq h \rightarrow 0$.

Notice that in the next result the hypothesis on continuity of $U \ni(x, y) \mapsto$ $D_{2} f(x, y) \in L_{\mathrm{c}}(B, E)$ is obsolete if $\operatorname{dim} B<\infty$.

TheOREM 7.3. Let a Fréchet space T, Banach spaces $B$ and $E$, an open set $U \subset T \times B$, a continuously differentiable map $f: U \rightarrow E$, and a zero $\left(t_{0}, x_{0}\right) \in U$ of $f$ be given. Assume that $D_{2} f\left(t_{0}, x_{0}\right): B \rightarrow E$ is bijective and that the map $U \ni$ $(t, x) \mapsto D_{2} f(t, x) \in L_{\mathrm{c}}(B, E)$ is continuous. Then there are open neighbourhoods $V$ of $t_{0}$ in $T$ and $W$ of $x_{0}$ in $B$ with $V \times W \subset U$ and a continuously differentiable map $g: V \rightarrow W$ with $g\left(t_{0}\right)=x_{0}$ and

$$
\{(t, x) \in V \times W: f(t, x)=0\}=\{(t, x) \in V \times W: x=g(t)\} .
$$

Proof. 1. (A fixed point problem) Choose an open neighbourhood $N_{T, 1}$ of $t_{0}$ and a convex open neighbourhood $N_{B}$ of $x_{0}$ in $B$ with $N_{T, 1} \times N_{B} \subset U$. The equation

$$
f(t, x)=f\left(t, x_{0}\right)+D_{2} f\left(t_{0}, x_{0}\right)\left[x-x_{0}\right]+R(t, x)
$$


defines a continuously differentiable map $R: N_{T, 1} \times N_{B} \rightarrow E$, with $R\left(t, x_{0}\right)=0$ for all $t \in N_{T, 1}$,

$$
D_{2} R(t, x)=D_{2} f(t, x)-D_{2} f\left(t_{0}, x_{0}\right) \quad \text { for all } t \in N_{T, 1} \text { and } x \in N_{B} \text {, }
$$

and, in particular, $D_{2} R\left(t_{0}, x_{0}\right)=0$. The map

$$
N_{T, 1} \times N_{B} \ni(t, x) \mapsto D_{2} R(t, x) \in L_{\mathrm{c}}(B, E)
$$

is continuous. In order to solve the equation $0=f(t, x),(t, x) \in N_{T, 1} \times N_{B}$, for $x$ as a function of $t$, observe that the previous equation is equivalent to

$$
0=f\left(t, x_{0}\right)+D_{2} f\left(t_{0}, x_{0}\right)\left[x-x_{0}\right]+R(t, x),
$$

or

$$
\begin{aligned}
x & =x_{0}+\left(D_{2} f\left(t_{0}, x_{0}\right)\right)^{-1}\left[-f\left(t, x_{0}\right)-R(t, x)\right] \\
& =x_{0}-\left(D_{2} f\left(t_{0}, x_{0}\right)\right)^{-1} f\left(t, x_{0}\right)-\left(D_{2} f\left(t_{0}, x_{0}\right)\right)^{-1} R(t, x) .
\end{aligned}
$$

The last expression defines a map $A: N_{T, 1} \times N_{B} \rightarrow B$ with $A\left(t_{0}, x_{0}\right)=x_{0}$, and for $(t, x) \in N_{T, 1} \times N_{B}, 0=f(t, x)$ if and only if $x=A(t, x)$. The map $A$ is continuously differentiable since the linear map $\left(D_{2} f\left(t_{0}, x_{0}\right)\right)^{-1}: E \rightarrow B$ is continuous, due to the open mapping theorem.

2. (Contraction) For all $t \in N_{T, 1}$ and for all $x, \widehat{x}$ in $N_{B}$,

$$
\begin{aligned}
|A(t, \widehat{x})-A(t, x)| & =\left|-\left(D_{2} f\left(t_{0}, x_{0}\right)\right)^{-1} R(t, \widehat{x})+\left(D_{2} f\left(t_{0}, x_{0}\right)\right)^{-1} R(t, x)\right| \\
& \leq\left|\left(D_{2} f\left(t_{0}, x_{0}\right)\right)^{-1}\right|\left|\int_{0}^{1} D_{2} R(t, x+s[\widehat{x}-x])[\widehat{x}-x] d s\right| .
\end{aligned}
$$

Let $\varepsilon=1 /\left(2\left|\left(D_{2} f\left(t_{0}, x_{0}\right)\right)^{-1}\right|\right)$. There are an open neighbourhood $N_{T, 2} \subset N_{T, 1}$ of $t_{0}$ and $\delta>0$ such that for all $t \in N_{T, 2}$ and all $x \in B$ with $\left|x-x_{0}\right| \leq \delta$,

$$
x \in N_{B} \quad \text { and } \quad\left|D_{2} R(t, x)\right|=\left|D_{2} R(t, x)-D_{2} R\left(t_{0}, x_{0}\right)\right|<\varepsilon .
$$

For all $x \neq \widehat{x}$ in $B$ with $\left|x-x_{0}\right| \leq \delta$ and $\left|\widehat{x}-x_{0}\right| \leq \delta$ and for all $s \in[0,1]$ it follows that $\left|x+s[\widehat{x}-x]-x_{0}\right| \leq \delta$, hence

$$
\left|D_{2} R(x+s[\widehat{x}-x]) \frac{1}{|\widehat{x}-x|}[\widehat{x}-x]\right|<\varepsilon,
$$

and thereby

$$
|A(t, \widehat{x})-A(t, x)| \leq \varepsilon|\widehat{x}-x|\left|\left(D_{2} f\left(t_{0}, x_{0}\right)\right)^{-1}\right|=\frac{1}{2}|\widehat{x}-x| .
$$

3. (Invariance) By continuity, there is an open neighbourhood $N_{T, 3} \subset N_{T, 2}$ of $t_{0}$ such that

$$
\left|A\left(t, x_{0}\right)-A\left(t_{0}, x_{0}\right)\right|<\frac{\delta}{4} \quad \text { for all } t \in N_{T, 3} .
$$


For all $t \in N_{T, 3}$ and $x \in B$ with $\left|x-x_{0}\right| \leq \delta$ this yields

$$
\begin{aligned}
\left|A(t, x)-x_{0}\right| & =\left|A(t, x)-A\left(t_{0}, x_{0}\right)\right| \\
& \leq\left|A(t, x)-A\left(t, x_{0}\right)\right|+\left|A\left(t, x_{0}\right)-A\left(t_{0}, x_{0}\right)\right| \\
& <\frac{1}{2}\left|x-x_{0}\right|+\frac{\delta}{4} \leq \frac{\delta}{2}+\frac{\delta}{4}=\frac{3 \delta}{4} .
\end{aligned}
$$

4. Set $V=N_{T, 3}, O_{B}=\left\{x \in B:\left|x-x_{0}\right|<\delta\right\}$, and

$$
M=\left\{x \in B:\left|x-x_{0}\right| \leq \frac{3 \delta}{4}\right\},
$$

and apply Theorem 7.2 to the restriction of $A$ to the set $V \times O_{B}$. This yields a continuously differentiable map $g: V \rightarrow B$ with $g(t)=A(t, g(t)) \in O_{B}$ for all $t \in V$. Using part 3, we get $\left|g(t)-x_{0}\right|<3 \delta / 4$ for all $t \in V$. Set

$$
W=\left\{x \in B:\left|x-x_{0}\right|<\frac{3 \delta}{4}\right\} .
$$

Then $g(V) \subset W$. From $g(t)=A(t, g(t))$ for all $t \in V$ we obtain $0=f(t, g(t))$ for these $t$. Conversely, if $0=f(t, x)$ for $(t, x) \in V \times W \subset V \times M$, then $x=A(t, x)$, hence $x=g(t)$. In particular, $x_{0}=g\left(t_{0}\right)$.

\section{REFERENCES}

[1] O. Diekmann And M. Gyllenberg, Equations with infinite delay: Blending the abstract with the concrete, J. Differential Equations 252 (2012), 819-851.

[2] O. Diekmann, S.A. van Gils, S.M. Verduyn Lunel and H.O. Walther, Delay Equations: Functional-, Complex- and Nonlinear Analysis, Springer-Verlag, 1995.

[3] H. GLÖCKNER, Implicit functions from topological vector spaces to Banach spaces, Israel J. Math. 155 (2006), 205-252.

[4] _ Finite order differentiability properties, fixed points and implicit functions over valued fields, arxiv.org/pdf/math/0511218, preprint, 59 pp., 2007.

[5] J.K. Hale and S.M. Verduyn Lunel, Introduction to Functional Differential Equations, Springer-Verlag, 1993.

[6] R.S. Hamilton, The inverse function theorem of Nash and Moser, Bull. Amer. Math. Soc. (N.S.) 7 (1982), 65-222.

[7] F. Hartung, T. Krisztin, H.O. Walther and J. Wu, Functional differential equations with state-dependent delays: Theory and applications, Handbook of Differential Equations, Ordinary Differential Equations, (A. Canada, P. Drabek, A. Fonda, eds), vol. 3, Elsevier Science B.V., North Holland, 2006, 435-545.

[8] Y. Hino, S. Murakami and T. Naito, Functional Differential Equations with Infinite Delay, Lecture Notes in Math., vol. 1473, Springer-Verlag, 1991

[9] J. Mallet-Paret, R.D. Nussbaum and P. Paraskevopoulos, Periodic solutions for functional differential equations with multiple state-dependent time lags, Topol. Methods Nonlinear Anal. 3 (1994), 101-162.

[10] W. Rudin, Functional Analysis, McGraw-Hill, 1973.

[11] K. Schumacher, Existence and continuous dependence for functional-differential equations with unbounded delay, Arch. Rational Mech. Anal. 67 (1978), 315-335. 
[12] T. Sengadir, Semigroups on Fréchet spaces and equations with infinite delay, Proc. Indian. Acad. Sci (Math. Sci.) 117 (2007), 71-84.

[13] J. SzILAsi AND R.L. Lovas, Some aspects of differential theories, Handbook of Global Analysis, (D. Krupka and D. Saunders, eds), Elsevier Science B.V., North Holland, 2007, 1071-1116.

[14] H.O. WALTHER, The solution manifold and $C^{1}$-smoothness of solution operators for differential equations with state dependent delay, J. Differential Equations 195 (2003), $46-65$.

[15] _ Smoothness properties of semiflows for differential equations with state dependent delay, J. Math. Sciences 124 (2004), 5193-5207.

[16] _ Differential equations with locally bounded delay, J. Differential Equations 252 (2012), 3001-3039.

[17] _ Evolution systems for differential equations with variable time lags, J. Math. Sciences 202 (2014), 911-933.

[18] _ Local invariant manifolds for delay differential equations with state space in $C^{1}\left((-\infty, 0], \mathbb{R}^{n}\right)$, Electron. J. Qual. Theory Differ. Equ., to appear.

Manuscript received May 28, 2015

accepted October 12, 2015

HANS-OtTo WALTHER

Mathematisches Institut

Universität Gießen

Arndtstr. 2

D-35392 Gießen, GERMANY

E-mail address: Hans-Otto.Walther@math.uni-giessen.de 